\title{
Non-Oberbeck-Boussinesq effects in turbulent thermal convection in ethane close to the critical point
}

\author{
Guenter Ahlers, ${ }^{1}$ Enrico Calzavarini, ${ }^{2}$ Francisco Fontenele Araujo, ${ }^{2}$ Denis Funfschilling, ${ }^{3}$ Siegfried Grossmann, ${ }^{4}$ \\ Detlef Lohse, ${ }^{2}$ and Kazuyasu Sugiyama ${ }^{2}$ \\ ${ }^{1}$ Department of Physics and iQCD, University of California, Santa Barbara, California 93106, USA \\ ${ }^{2}$ Department of Applied Physics and J. M. Burgers Centre for Fluid Dynamics, University of Twente, \\ 7500 AE Enschede, The Netherlands \\ ${ }^{3}$ LSGC CNRS_GROUPE ENSIC, Boîte Postale 451, 54001 Nancy Cedex, France \\ ${ }^{4}$ Department of Physics, Philipps-University of Marburg, Renthof 6, D-35032 Marburg, Germany
}

(Received 26 November 2007; published 8 April 2008)

\begin{abstract}
As shown in earlier work [Ahlers et al., J. Fluid Mech. 569, 409 (2006)], non-Oberbeck-Boussinesq (NOB) corrections to the center temperature in turbulent Rayleigh-Bénard convection in water and also in glycerol are governed by the temperature dependences of the kinematic viscosity and the thermal diffusion coefficient. If the working fluid is ethane close to the critical point, the origin of non-Oberbeck-Boussinesq corrections is very different, as will be shown in the present paper. Namely, the main origin of NOB corrections then lies in the strong temperature dependence of the isobaric thermal expansion coefficient $\beta(T)$. More precisely, it is the nonlinear $T$ dependence of the density $\rho(T)$ in the buoyancy force that causes another type of NOB effect. We demonstrate this through a combination of experimental, numerical, and theoretical work, the last in the framework of the extended Prandtl-Blasius boundary-layer theory developed by Ahlers et al. as cited above. The theory comes to its limits if the temperature dependence of the thermal expension coefficient $\beta(T)$ is significant. The measurements reported here cover the ranges $2.1 \leqq \operatorname{Pr} \lesssim 3.9$ and $5 \times 10^{9} \leqq \operatorname{Ra} \lesssim 2 \times 10^{12}$ and are for cylindrical samples of aspect ratios 1.0 and 0.5 .
\end{abstract}

DOI: 10.1103/PhysRevE.77.046302

PACS number(s): 47.55.pb, 47.27.te

\section{INTRODUCTION}

Fluid motion in the presence of temperature gradients is an important phenomenon in nature and industrial processes. Among the many examples are oceanic streams, cloud motions, and gusts of wind that can be felt on a human scale. The paradigmatical system for such thermally driven flows is the Rayleigh-Bénard (RB) setup: a fluid-filled container heated from below and cooled from above. In this classical problem, the flow is determined by the scale and geometry of the container, the material properties of the working fluid, and the top-down temperature difference $\Delta \equiv T_{b}-T_{t}>0$. In the last two decades, considerable progress has been achieved in our understanding of global and local properties and the flow organization of turbulent RB convection, through a combination of experimental (see, e.g., [1-24]), numerical (see, e.g., [25-35]), and theoretical work (see, e.g., [36-38]).

The temperature difference in a RB cell can be increased in a controlled way. However, in principle the transport coefficients of the fluid can depend on the local temperature and density and thus vary across the height $L$ of the container. Since space-dependent properties of this kind are undesirable in the first instance, one tends to restrict the convection regime to sufficiently small intervals of $\Delta$. But even so, further simplifications are progressively required in the analysis of RB convection. In this spirit, a standard approximation due to Oberbeck [39] and Boussinesq [40] assumes the following (see also [41,42]).

(OB1) The dynamic viscosity $\eta$, the thermal conductivity $\Lambda$, the thermal expansivity $\beta$, and the isobaric specific heat $c_{P}$ are constant throughout the fluid.
(OB2) Density variations are taken into account only in the buoyancy force term.

(OB3) The temperature dependence of the density $\rho$ is linearized in the buoyancy force as

$$
\rho(T)=\rho_{m}-\rho_{m} \beta_{m}\left(T-T_{m}\right),
$$

where $T_{m} \equiv\left(T_{b}+T_{t}\right) / 2$ is the arithmetic mean temperature between the plates and $X_{m}=X\left(T_{m}\right)$ denotes the fluid property $X$ evaluated at $T_{m}$.

Next to the aspect ratio, within the OB approximation two dimensionless parameters characterize the RB flow: The Prandtl number $\operatorname{Pr} \equiv \nu_{m} / \kappa_{m}$ follows from the ratio between the kinematic viscosity $\nu_{m} \equiv \eta_{m} / \rho_{m}$ and the thermal diffusivity $\kappa_{m} \equiv \Lambda_{m} /\left(\rho_{m} c_{P, m}\right)$. The dimensionless thermal driving can be conveniently represented by the Rayleigh number Ra $\equiv \beta_{m} g L^{3} \Delta /\left(\nu_{m} \kappa_{m}\right)$, where $g$ denotes the gravitational acceleration.

The manner in which high Rayleigh numbers are achieved is crucial for the emergence of non-Oberbeck-Boussinesq (NOB) effects. Since turbulent convection may involve spatiotemporal changes in the fluid properties, considerable efforts have been devoted to the identification of the dominating sources of NOB effects. In liquids like water [43] and glycerol $[44,45]$, for example, NOB effects are dominated by deviations from assumption $\mathrm{OB} 1$ since the viscosity strongly decreases with increasing temperature. On the other hand, when the working fluid is gaseous ethane [46], deviations from assumptions OB1 and OB2 lead to NOB effects stronger than those in the aforementioned liquids.

In the present study, we shall focus on deviations from assumption $\mathrm{OB} 3$ by considering the nonlinear temperature 
dependence of the buoyancy force. In particular, ethane close to its critical point [47] is chosen as the working fluid, and the temperature $T_{c}$ in the center of the container is measured as indicator of NOB effects.

There are two possibilities to characterize the physics beyond condition OB3 as described by Eq. (1), if the density $\rho(T)$ has a strong nonlinear $T$ dependence. First, one can introduce a $T$-dependent thermal expansion function $\hat{\beta}(T)$ instead of $\beta_{m}$, defined in terms of the density $\rho(T)$ by

$$
\rho(T) \equiv \rho_{m}-\rho_{m}\left(T-T_{m}\right) \hat{\beta}(T) .
$$

Second, one refers to the common isobaric thermal expansion coefficient $\beta(T)$, defined as usual by

$$
\beta(T) \equiv-\left.\frac{1}{\rho(T)} \frac{\partial \rho(T)}{\partial T}\right|_{P},
$$

which now is temperature dependent. The two are related by $\beta(T)=-\partial \ln \left[1-\left(T-T_{m}\right) \hat{\beta}(T)\right] / \partial T$. Under the condition OB3 of linear $T$ dependence of $\rho$, the thermal expansion function is constant, $\hat{\beta}=\beta_{m}$, while the expansion coefficient $\beta(T)$ is given by $\beta(T)=\beta_{m} /\left[1-\left(T-T_{m}\right) \beta_{m}\right]$, still depending on temperature. Of course, all three coincide at $T_{m}$. An advantage of considering the thermal expansion coefficient $\beta(T)$ is that it is a well-defined thermodynamic derivative. The advantage of $\hat{\beta}(T)$, on the other hand, is that it immediately reflects the nonlinear $T$ dependence of $\rho(T)$. Also $\beta(T)$ refers to a single thermodynamic state and describes the local $T$ slope on an isobar (normalized by the local density), while $\hat{\beta}(T)$ refers to a pair of states, namely, to the reference state $T_{m}$ in addition to $T$, and describes the secant to the $\rho(T)$ curve (normalized by the reference density). $\hat{\beta}(T)$ will therefore in general vary less with $T$ than does $\beta(T)$.

It will turn out that it is the significantly different $T$ dependence of $\hat{\beta}(T)$ [or $\beta(T)$ ] on the two sides of the critical isochore of ethane that leads to opposite shifts of the center (bulk) temperature $T_{c}$, yielding $T_{c}<T_{m}$ on the gaslike (i.e., high-temperature; see Fig. 1 below) side and $T_{c}>T_{m}$ on the liquidlike (low-temperature) side. On the gaslike side $\hat{\beta}(T)$ increases from bottom to top and on the liquidlike side it decreases.

Our approach consists of three stages: boundary-layer (BL) theory, experiments, and direct numerical simulations (DNSs). First, we address in Sec. II an extension of boundary-layer theory that considers deviations from assumptions OB1 and OB2. Even though the buoyancy force is not included in the BL equations (only the longitudinal momentum is taken into account here), we compute $T_{c}(\Delta)$ for several pressures $P_{m}$. Then, experimental measurements of $T_{c}(\Delta)$ are presented in Sec. III B and compared with BL results in Sec. IV. Given the significant discrepancies between some of them, we address in Sec. V direct numerical simulations that explicitly consider deviations from assumption OB3. In particular, for $T_{m}=27{ }^{\circ} \mathrm{C}$ and $P_{m}=51.72 \mathrm{bar}$, it is shown that NOB effects in ethane are dominated by the nonlinear dependence of the buoyancy force on temperature. Finally, our conclusions are summarized in Sec. VI. Appen-

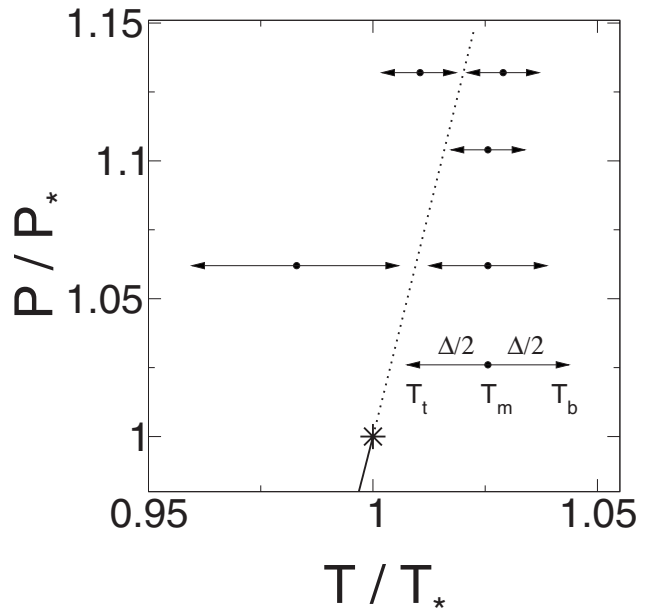

FIG. 1. Pressure-temperature plane in reduced units. Star: critical point of ethane $\left(T_{*}=32.18{ }^{\circ} \mathrm{C}, P_{*}=48.718\right.$ bars $)$. Solid line: liquid-vapor coexistence curve. Dotted line: critical isochore. The horizontal arrows show the maximum temperature intervals $\Delta$. From bottom to top they correspond to (i) $P_{m}=49.98$ bars $=1.026 P_{*}$, centered at $T_{m}=40.00{ }^{\circ} \mathrm{C}=1.025 T_{*}$, (ii) $P_{m}=51.72$ bars $=1.062 P_{*}$, centered at $T_{m}=40.00{ }^{\circ} \mathrm{C}$ (right of critical isochore) and at $T_{m}=27.00{ }^{\circ} \mathrm{C}=0.983 T_{*}$ (left of critical isochore), and (iii) $P_{m}=53.78$ bars $=1.104 P_{*}$, centered at $T_{m}=40.00{ }^{\circ} \mathrm{C}$, and (iv) $P_{m}$ $=55.17$ bar $=1.132 P_{*}$, centered at $T_{m}=41.00{ }^{\circ} \mathrm{C}=1.029 T_{*}$ (right of critical isochore) and at $T_{m}=35.00{ }^{\circ} \mathrm{C}=1.009 T_{*}$ (left of critical isochore).

dixes $\mathrm{A}$ and $\mathrm{B}$ are devoted to the derivation of the boundarylayer equations with variable transport coefficients and Appendix C compiles the Nusselt number corrections for the real and various hypothetical ethanelike fluids in a table.

\section{BOUNDARY-LAYER THEORY}

A central aspect in Rayleigh-Bénard convection is the understanding of the boundary layers formed along the bottom and top plates. Though they preserve a laminar character for $\mathrm{Ra} \leq 10^{12}$, their instabilities impact the Nusselt number $\mathrm{Nu}$ (the effective heat flux relative to thermal conduction $\left.\Lambda_{m} \Delta / L\right)[48,49]$. As reported in Refs. [43,44,46], BL flows of this nature are significantly influenced by the coupling between the fluid properties and the temperature gradient across the container. In particular, it was shown that NOB effects on $T_{c}$ can be reasonably described by extending the Prandtl-Blasius boundary-layer theory [50,51]. Next we review such an extension and, further, assess its intrinsic limitations.

Assume that the density $\rho$, the temperature $T$, and the velocity $\mathbf{u}$ are stationary fields, which depend only on the longitudinal $x$ and transverse $z$ coordinates. Then, under the boundary-layer approximation, we write the continuity and the $x$ momentum equations as (see also Appendix A 1)

$$
\frac{\partial}{\partial x}\left\{\rho u_{x}\right\}+\frac{\partial}{\partial z}\left\{\rho u_{z}\right\}=0
$$




$$
\rho\left\{u_{x} \frac{\partial u_{x}}{\partial x}+u_{z} \frac{\partial u_{x}}{\partial z}\right\}=\frac{\partial}{\partial z}\left\{\eta \frac{\partial u_{x}}{\partial z}\right\} .
$$

Here $z$ measures the vertical distance from the bottom or top plate, respectively, and the velocity components at $z=0$ are subject to no-slip boundary conditions $u_{x}(x, 0)=0$ and $u_{z}(x, 0)=0$. Moreover, in the bulk of the flow, $u_{x}$ is supposed to match the large-scale wind velocity $U_{c}$ in the center (bulk) of the RB sample [52], i.e., $u_{x}(x, \infty)=U_{c}$. Note that within the BL theory we cannot calculate $U_{c}$; here we only have to assume that it is the same close to the top and the bottom BLs, which is supported by our numerical simulations reported in Sec. V.

In the same spirit, the temperature field $T(x, z)$ is governed by (cf. Appendix A 2)

$$
\frac{\gamma}{\rho c_{P}} \frac{\partial}{\partial z}\left\{\Lambda \frac{\partial T}{\partial z}\right\}=u_{x} \frac{\partial T}{\partial x}+u_{z} \frac{\partial T}{\partial z}+\frac{\gamma-1}{\beta}\left\{\frac{\partial u_{x}}{\partial x}+\frac{\partial u_{z}}{\partial z}\right\},
$$

where $\gamma \equiv c_{P} / c_{V}$ is the ratio between the isobaric and isochoric specific heats and $\beta=-\rho^{-1} \partial \rho / \partial T$ denotes the isobaric thermal expansion coefficient. At the plates $T(x, 0)=T_{b, t}$ and in the bulk (center) of the flow $T(x, \infty)=T_{c}$.

The coupling between the bottom and top boundary layers is determined (cf [43]) by the heat fluxes $Q_{b, t}$ through the plates, considered to be equal:

$$
Q_{b}=-\left.\Lambda_{b} \frac{\partial T}{\partial z}\right|_{b}=-\left.\Lambda_{t} \frac{\partial T}{\partial z}\right|_{t}=Q_{t} .
$$

This condition establishes an implicit dependence of the center temperature $T_{c}$ on the heat fluxes $Q_{b}=Q_{t}=Q$. Note again that both the dynamic viscosity $\eta$ and the heat conductivity $\Lambda$ depend on both temperature and density, i.e., $\eta(T, \rho)$ and $\Lambda(T, \rho)$. Before addressing the technicalities around the (numerical) integration of Eqs. (4)-(7), we shall benefit from a key argument in boundary-layer theory: Prandtl's self-similar ansatz.

\section{A. Self-similarity}

Because of the $y$ independence, assumed in the Prandtl BL theory, the boundary layer flow is mathematically a twodimensional (2D) flow. Therefore the system of partial differential equations (4)-(6) for the BL flow can be reduced to ordinary differential equations (ODEs) by introducing a stream function $\Psi$. We do this differently from the usual procedure by including in its definition the density in order to automatically satisfy the continuity equation by construction:

$$
\begin{gathered}
\tilde{\rho} u_{x}=\frac{\partial \Psi}{\partial z}, \\
\tilde{\rho} u_{z}=-\frac{\partial \Psi}{\partial x},
\end{gathered}
$$

where $\tilde{\rho} \equiv \rho / \rho_{m}$ is the density nondimensionalized with $\rho_{m}$ $=\rho\left(T_{m}, P_{m}\right)$. Clearly the continuity equation automatically follows from (8) and (9). Next (see Appendix B), we may introduce a self-similarity variable $\widetilde{Z} \equiv z / \ell_{c}(x)$ and a similarity function $\widetilde{\Psi}(\tilde{Z})=\Psi(x, z) /\left(\ell_{c}(x) U_{c}\right)$, such that $\ell_{c}(x)$ $=\sqrt{x \nu_{m} / U_{c}}$. Thus the velocity components are

$$
u_{x}=U_{c} \frac{\widetilde{\Psi}^{\prime}}{\tilde{\rho}}, \quad u_{z}=\frac{\nu_{m}}{2 \ell_{c}}\left(\tilde{Z} \frac{\widetilde{\Psi}^{\prime}}{\tilde{\rho}}-\frac{\tilde{\Psi}}{\tilde{\rho}}\right),
$$

with boundary conditions $\tilde{\Psi}(0)=0=\widetilde{\Psi}^{\prime}(0)$ and $\widetilde{\Psi}^{\prime}(\infty)=\widetilde{\rho}_{c}$.

In terms of (10), the viscous BL equation (5) can be written as

$$
\begin{aligned}
0= & \tilde{\eta} \widetilde{\Psi}^{\prime \prime \prime}+\left(\frac{1}{2} \widetilde{\Psi}+\widetilde{\eta}^{\prime}-2 \frac{\widetilde{\rho}^{\prime}}{\widetilde{\rho}} \tilde{\eta}\right) \widetilde{\Psi}^{\prime \prime} \\
& +\left\{-\frac{1}{2} \frac{\widetilde{\rho}^{\prime}}{\widetilde{\rho}} \widetilde{\Psi}+\left[2\left(\frac{\widetilde{\rho}^{\prime}}{\widetilde{\rho}}\right)^{2}-\frac{\widetilde{\rho}^{\prime \prime}}{\widetilde{\rho}}\right] \widetilde{\eta}-\frac{\widetilde{\rho}^{\prime}}{\widetilde{\rho}} \widetilde{\eta}^{\prime}\right\} \widetilde{\Psi}^{\prime} .
\end{aligned}
$$

Here $\widetilde{\eta} \equiv \eta / \eta_{m}$ is the dimensionless viscosity, whose $\widetilde{Z}$ dependence $\widetilde{\eta}^{\prime}$ is given by

$$
\tilde{\eta}^{\prime}=\left(\frac{\partial \widetilde{\eta}}{\partial \widetilde{\Theta}}\right)_{\widetilde{\rho}} \widetilde{\Theta}^{\prime}+\left(\frac{\partial \tilde{\eta}}{\partial \widetilde{\rho}}\right)_{\tilde{\Theta}} \widetilde{\rho}^{\prime},
$$

where $\widetilde{\Theta} \equiv\left(T-T_{t}\right) / \Delta$ denotes the dimensionless temperature.

Next, assuming that the pressure $P_{m}$ is constant throughout the fluid, one finds

$$
\tilde{\rho}^{\prime}=-\tilde{\rho} \widetilde{\beta} \widetilde{\Theta}^{\prime},
$$

with $\widetilde{\beta} \equiv \beta \Delta$. The boundary conditions at the respective walls are $\widetilde{\rho}(0)=\widetilde{\rho}_{b, t}, \widetilde{\rho}^{\prime}(0)=-\widetilde{\rho}_{b, t} \widetilde{\beta}_{b, t} \widetilde{\Theta}_{b, t}^{\prime}$, and $\widetilde{\rho}(\infty)=\widetilde{\rho}_{c}$.

Finally, we also write the temperature Eq. (6) in selfsimilar form as (see Appendix B 2)

$$
\widetilde{\Lambda} \widetilde{\Theta}^{\prime \prime}+\left(\frac{1}{2} \widetilde{c}_{P} \operatorname{Pr} \widetilde{\Psi}+\widetilde{\Lambda}^{\prime}\right) \widetilde{\Theta}^{\prime}=0,
$$

where $\tilde{\Lambda} \equiv \Lambda / \Lambda_{m}$ and $\widetilde{c}_{P} \equiv c_{P} / c_{P, m}$. Equation (13) is subject to $\widetilde{\Theta}(0)=\widetilde{\Theta}_{b, t}$ and $\widetilde{\Theta}(\infty)=\widetilde{\Theta}_{c}$.

\section{B. Results}

The coupled ODEs (11)-(13) with the corresponding boundary conditions and the heat-flux conservation (7) are solved numerically with a shooting method [53]. The integration domain is restricted to $\Delta$ intervals where the transport properties are concave or convex functions of the temperature. In particular, we have chosen ethane as the working fluid since its properties are known very well [47], even close to its critical point $\left(T_{*}, P_{*}, \rho_{*}\right)$ (see Fig. 1). All material properties $\eta, \Lambda, \rho, \beta$, and $c_{p}$ are implemented in their full dependence on $T$ In this manner, the computation of temperature and density profiles does not involve any fit parameter.

\section{Vertical profiles}

An insight into the structure of the BLs can be achieved by studying typical profiles along the $z$ direction. To describe 
(1)

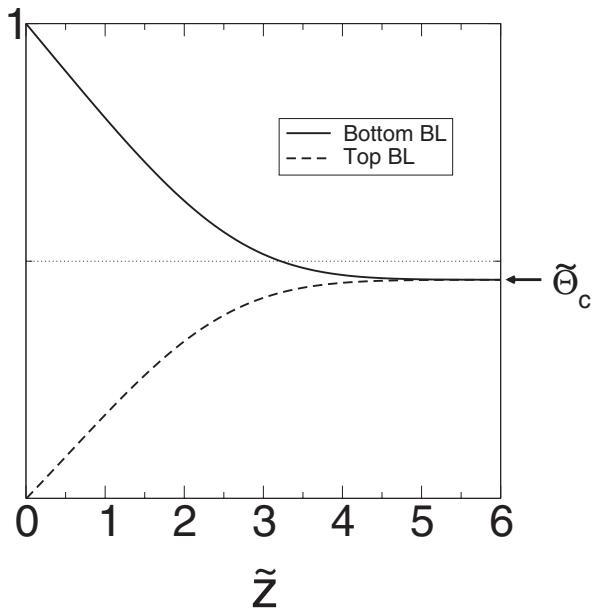

(a)

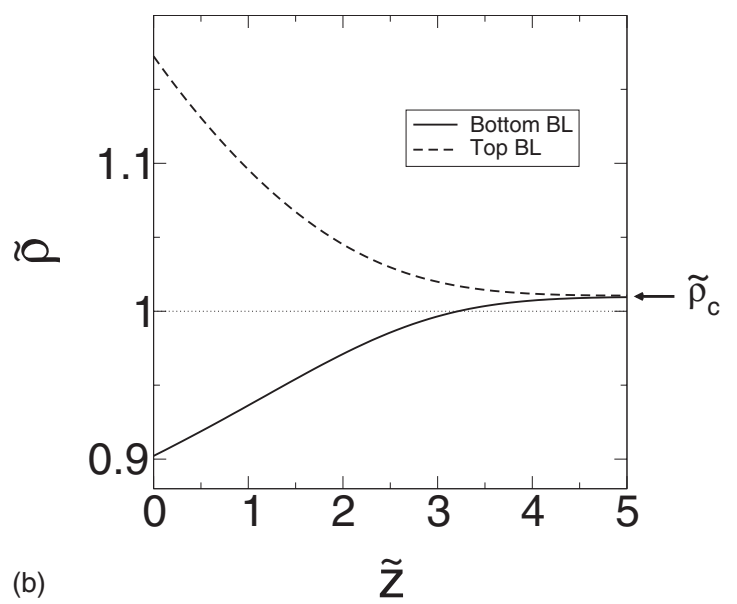

FIG. 2. (a) Temperature and (b) density profiles at $P_{m}$ $=0.849 P_{*}, T_{m}=40{ }^{\circ} \mathrm{C}$, and $\Delta=15 \mathrm{~K}$. The thermal slope thicknesses at the bottom or top $\lambda_{b, t}^{\mathrm{sl}} / L=a_{b, t} / \sqrt{\nu_{m} / L U_{c}}$ are seen to have prefactors of about $a_{b} \approx 2.8$ and $a_{t} \approx 2.5$, i.e., $\lambda_{b}^{\mathrm{sl}}>\lambda_{t}^{\mathrm{sl}}$.

them, let us consider a representative case in which the pressure is fixed at $P_{m}=0.849 \cdot P_{*}$, the mean temperature at $T_{m}$ $=40{ }^{\circ} \mathrm{C}$, and the thermal difference between the plates at $\Delta=15 \mathrm{~K}$.

In Fig. 2, the temperature $\widetilde{\Theta}$ and density $\widetilde{\rho}$ are plotted as functions of the similarity variable $\tilde{Z}$. As shown in Fig. 2(a), the center temperature $\widetilde{\Theta}_{c}$ is smaller than the mean temperature $\widetilde{\Theta}_{m}=0.5$, clearly indicating a top-down symmetry breaking. Such symmetry breaking is also reflected in the density profiles shown in Fig. 2(b), since the center density $\widetilde{\rho}_{c}$ is larger than the mean density $\widetilde{\rho}_{m}=1$. Notwithstanding the pronounced curvatures in $\widetilde{\Theta}(\widetilde{Z})$ and $\widetilde{\rho}(\widetilde{Z})$, we shall restrict our attention to the asymptotic value $\widetilde{\Theta}_{c}$ as a convenient indicator of NOB effects.

\section{Center temperature}

To compute the difference $T_{c}-T_{m}$ as a function of $\Delta$, we have chosen a particular set of isobars in the phase diagram of ethane. As shown in Fig. 1, our selection of $\Delta$ intervals falls into two classes: (i) those intervals centered at $T_{m}$ $=27{ }^{\circ} \mathrm{C}$ and (ii) those centered at $T_{m}=40{ }^{\circ} \mathrm{C}$.
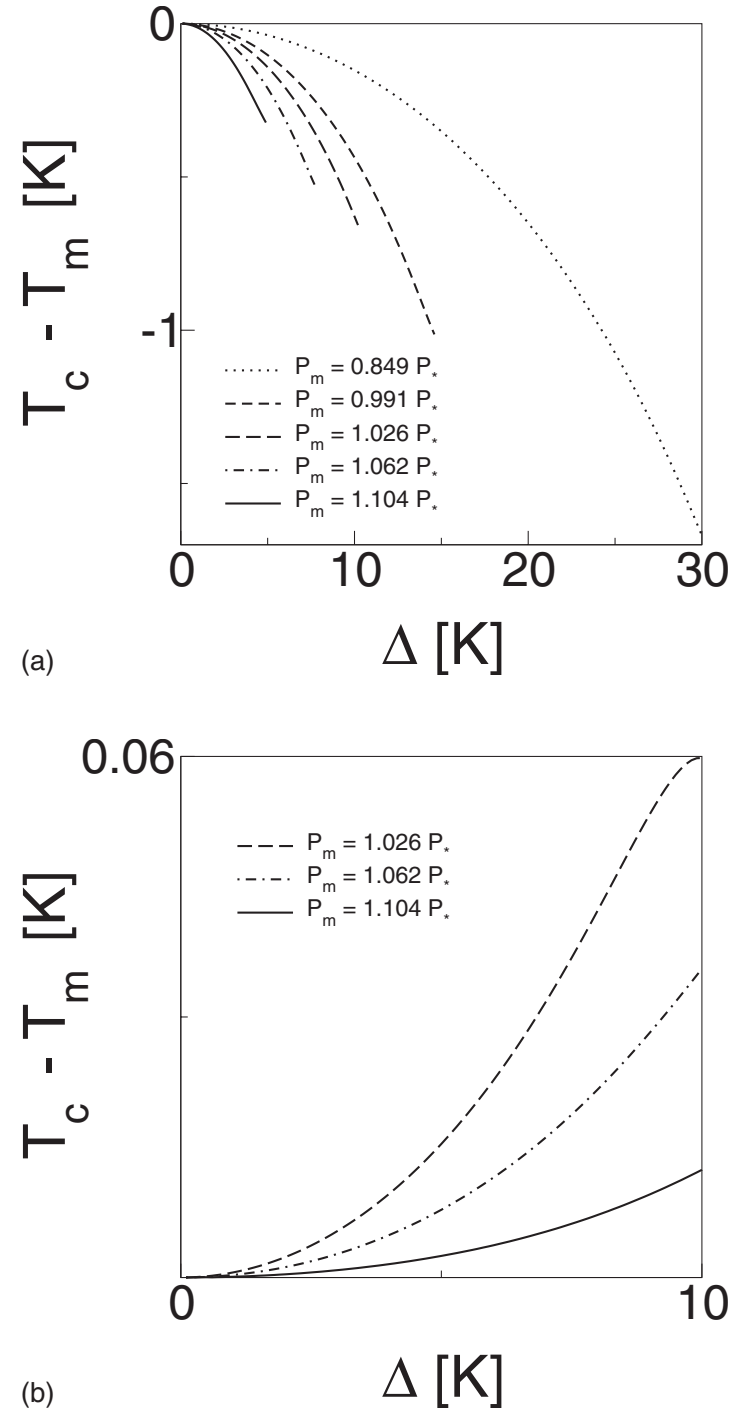

FIG. 3. Deviation $T_{c}-T_{m}$ as function of $\Delta$, for $T_{m}=$ (a) 40 and (b) $27{ }^{\circ} \mathrm{C}$.

As for the latter (the more gaseous case), Fig. 3(a) shows that the center temperature is a decreasing function of $\Delta$. The top-down symmetry of the flow is broken in such a way that the top boundary layer tends to become thinner than its bottom counterpart, eventually leading to a temperature reduction in the center of the flow. Though this result has been originally reported and explained already in Ref. [46], we briefly mention it here for completeness of discussion.

Focusing now on the class of $\Delta$ intervals centered at $T_{m}$ $=27{ }^{\circ} \mathrm{C}$, Fig. 3(b) shows that the center temperature becomes larger than the mean temperature between the plates. This NOB effect is different from what we found in Ref. [46] and repeated in Fig. 3(a), where we have focused on the more gaslike case.

To understand this we argue that the intervals under consideration (centered at $T_{m}=27{ }^{\circ} \mathrm{C}$ ) now correspond to a region of the phase diagram where the material properties of ethane behave more similarly to those of the liquid phase. NOB effects in classical liquids (such as water and glycerol) were already discussed in Refs. [43-45]. One of our aims in 


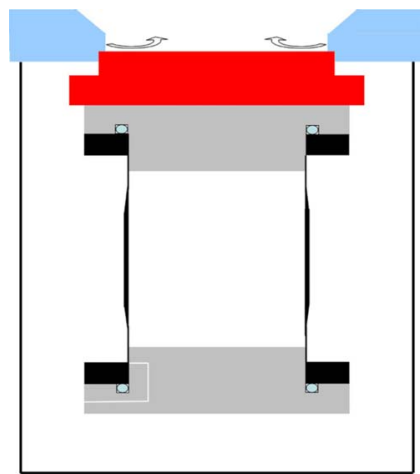

FIG. 4. (Color online) Schematic diagram (approximately to scale for $\Gamma=1$ ) of the high-pressure sample cell surrounded by the can containing ambient air and foam.

the present work is to further assess the differences in the NOB effects between the more liquidlike versus the more gaslike fluids (see Sec. III). It will turn out that this will show us the limitations of boundary-layer theory (see Sec. IV). To this end, we first consider now additional experimental details for determination of the $\Delta$ dependence of the center temperature $T_{c}(\Delta)$.

\section{EXPERIMENT}

\section{A. Apparatus}

The apparatus was described in detail before in Ref. [54], where a schematic diagram is shown in Fig. 2. Here we give a brief description and details specific to the present highpressure sample cell shown in Fig. 4. Working from the inside out, the sample cell was surrounded by a can containing ambient air. The air space inside the can was filled with low-density open-pore foam to prevent convection outside the sample. The maximum possible diameter of the sample top plate was $10 \mathrm{~cm}$, allowing for inside sample diameters typically up to about $D=8 \mathrm{~cm}$. The entire apparatus was of sufficient length to accommodate a sample with $L \simeq 16 \mathrm{~cm}$ $(\Gamma \simeq 0.5)$. Heat was applied at the sample bottom by a metalfilm heater covering the entire active bottom-plate area uniformly.

The top plate was cooled by a circulating water bath. The water was cooled by passing through a heat exchanger external to the main apparatus, which in turn was cooled by a separate water circuit driven by a Neslab or Lauda refrigerated circulator with a temperature stability of $0.01{ }^{\circ} \mathrm{C}$. Just before entering the apparatus, the water was heated by a heater consisting of about $25 \mathrm{~m}$ of Teflon-insulated AWG30 (0.5 mm diameter) copper wire, stuffed into the inlet line and thus immersed in the water. The large contact area between the water and the heater wire provided excellent heat exchange and uniform heating of the water. The heater was computer controlled in a feedback loop with a thermometer located in the top plate of the sample cell. The bathtemperature stability achieved in this way was a few tenths of a millikelvin. The water entered the bottom center of the apparatus, flowed upward through an annular channel around the can, and was distributed over the top plate by a set of jets. With this arrangement the entire can was kept at the top-plate temperature, and parasitic heat loss from the sidewall and the bottom plate due to conduction through the air and foam as well as by radiation was reduced to a level that was negligible compared to the heat transport by the convecting fluid.

After cooling the top plate the water returned through an annular channel located just outside of and mildly insulated from the incoming channel. Since the water, while cooling the top plate, was never heated by more than a few millikelvins, the returning water provided an excellent adiabatic thermal shield at the top-plate temperature, thus stabilizing the interior temperatures and preventing significant variations in time of the parasitic heat losses from the bottom plate. The entire apparatus sat on a chlorinated poly(vinyl chloride) (CPVC) base plate with appropriate channels and feedthroughs to accommodate the water circuit, the electrical leads, and the fill capillary going to the sample.

One of two high-pressure sample cells (see Fig. 4) was installed inside the can. It had the shape of a cylinder with $D=7.63 \mathrm{~cm}$. One cell had an internal length $L=7.62 \mathrm{~cm}$, corresponding to an aspect ratio $\Gamma \equiv D / L=1.00$. Another one had $L=15.24 \mathrm{~cm}$, yielding $\Gamma=0.500$. The top and bottom plates consisted of thick copper disks. Each of the two plates had an anvil, $1.59 \mathrm{~cm}$ thick, of diameter essentially equal to $D$, that was a close slide fitted in the type-4340 steel sidewall. After machining, the sidewall was heat treated at $830{ }^{\circ} \mathrm{C}$ and oil quenched. This procedure is expected to lead to a tensile strength of about 13 kbars. The sidewall had a flange at each end, of thickness $0.95 \mathrm{~cm}$. Each flange was bolted (not shown in the figure) and O-ring sealed to one of the copper end plates. A top and bottom thin section of the sidewall had a thickness of $0.051(0.076) \mathrm{cm}$ for the $\Gamma=1$ $(\Gamma=0.5)$ cell. This thin section overlapped the copper anvils and extended into the sample region by $0.95 \mathrm{~cm}$. Connecting the thin section was a central section of wall thickness 0.15 $\mathrm{cm}$ that provided enhanced strength; since the turbulent system contained only a very small thermal gradient in its center, the thicker wall section did not significantly enhance the wall heat transport. The sample entered the bottom copper plate through a capillary from the side, and then proceeded through a very small hole (shown in white on the left side of the figure) into the gap between the bottom-plate anvil and the sidewall.

The sample was connected to a manifold through a capillary. Also connected to the manifold was a separate pressureregulation volume of $600(1000) \mathrm{cm}^{3}$ in the case of the $\Gamma$ $=1(\Gamma=0.5)$ cell that could be heated above the ambient temperature by a heater wrapped around its outside. The temperature of this "hot volume" was controlled in a feedback loop with a pressure gage [55]. The pressure stability typically was better that 1 millibar. The entire system was designed to safely withstand pressures up to 60 bars. For the pressure measurements we used a Paroscientific model 745 pressure standard with an accuracy of $80 \mathrm{ppm}$ (about 6 mbars) and a resolution of 1 ppm (about $70 \mu$ bars) at full scale.

A substantial fraction of the heat current passed through the sidewall. This current was measured for the evacuated cell and subtracted from all other measurements; but, as was 
recognized some time ago $([56,57])$, this is not an adequate procedure because of the height-dependent temperature gradients that prevail in the wall when the cell contains turbulently convecting fluid. We did not attempt a correction for this nonlinear sidewall effect in the present case because we do not believe that a reliable correction is possible when the sidewall conductance is large. For this reason our values of $\mathrm{Nu}$ under $\mathrm{OB}$ conditions are about 25\% larger than other measurements at similar Prandtl numbers [16]. However, we believe that the deviations of $\mathrm{Nu}$ and of $T_{c}$ from their Boussinesq values, which (as we shall see below in Sec. III B 2) depend primarily on the nature of the top and bottom boundary layers rather than on the fluid interior, were obtained reliably.

The top and bottom temperatures $T_{t}$ and $T_{b}$ were determined from the average of six thermistors embedded close to the fluid in each of the top and bottom plates [58]. These thermistors were calibrated against a platinum-resistance thermometer purchased from Hart Scientific. This thermometer was supplied with a calibration, accurate to $\pm 7 \mathrm{mK}$, on the ITS90 temperature scale. The average temperature readings were used to obtain $\Delta=T_{b}-T_{t}$ and $T_{m}=\left(T_{t}+T_{b}\right) / 2$. Small corrections for the temperature gradients in the copper plates were applied. The center temperature $T_{c}$ was taken to be the average of the temperatures measured with eight thermistors attached to the outside of the sidewall at the horizontal midplane, equally spaced in the azimuthal direction (see, for instance, Ref. [22] or [59]).

In order to obtain an estimate of the $\mathrm{OB}$ values of the Nusselt numbers, a power law $\mathrm{Nu}_{\mathrm{OB}}=N_{0} R^{\gamma_{\text {eff }}}$ was fitted to the Nusselt-number measurements at relatively small $\Delta$ where $\beta_{m} \Delta \lesssim 0.05$, adjusting $\gamma_{\text {eff }}$ and $N_{0}$. Such fits yielded values of $\gamma_{\text {eff }}$ close to 0.30 . All the measured values of $\mathrm{Nu}$ regardless of $\beta_{m} \Delta$ were then divided by the power-law value at the measured Rayleigh numbers to give $\mathrm{Nu} / \mathrm{Nu}_{\mathrm{OB}}$ at all $\Delta$.

All measurements reported here were made with many values of $\Delta$ at each of a few constant values of $T_{m}$ and $P$. In both cells we used ethane at elevated pressures as the fluid. The thermophysical properties were calculated from the formulas given in Ref. [47]. For extensive discussions of the uncertainties of these properties we refer to that paper. It is difficult to determine the absolute errors for the Rayleigh and Nusselt numbers that result from property uncertainties, but we expect that an estimate of a few percent is not unreasonable. Since in the present paper we are concerned only with the ratios $\mathrm{Nu} / \mathrm{Nu}_{\mathrm{OB}}$, and since all data are taken as a function of $\Delta$ at a given mean temperature and pressure and evaluated at the same $P$ and $T_{m}$, property errors cancel to a very large extent.

\section{B. Results}

\section{The Nusselt number $\mathrm{Nu}(\mathrm{Ra})$}

As indicated above, we do not regard the results for $\mathrm{Nu}(\mathrm{Ra})$ to be very accurate because of unknown effects due to the relatively large wall conductivity. Nonetheless we show the results for $\Gamma=1$ at several $T_{m}$ and $P$ in Fig. 5 on logarithmic scales. Over a wide range of $\mathrm{Ra}$ one sees that they are a few percent higher than the results from Refs. [6]

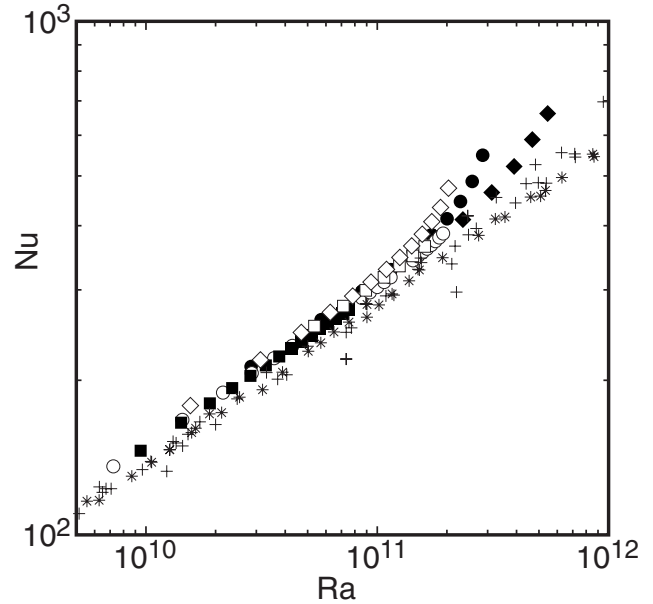

FIG. 5. Nusselt number $\mathrm{Nu}$ as a function of the Rayleigh number Ra for $\Gamma=1.00$. Solid circles: $P=51.72$ bars and $T_{m}=40{ }^{\circ} \mathrm{C}$ $(\mathrm{Pr}=2.58)$. Open circles: $P=51.72$ bars and $T_{m}=27{ }^{\circ} \mathrm{C}(\mathrm{Pr}=2.99)$. Solid squares: $P=51.72$ bars and $T_{m}=24{ }^{\circ} \mathrm{C}(\operatorname{Pr}=2.71)$. Open squares: $P=51.72$ bars and $T_{m}=31{ }^{\circ} \mathrm{C}(\operatorname{Pr}=3.85)$. Solid diamonds: $P=53.79$ bars and $T_{m}=40{ }^{\circ} \mathrm{C} \quad(\operatorname{Pr}=3.79)$. Open diamonds: $P$ $=50.00$ bars and $T_{m}=40{ }^{\circ} \mathrm{C}(\operatorname{Pr}=2.09)$. Stars from Ref. [6] for $\Gamma$ $=0.5$ after correction for sidewall effects. Pluses from Ref. [7] for $\Gamma=0.5$.

and [7], and we attribute this to the influence of the sidewall conductivity on our data. At the largest $\mathrm{Ra}$ our results increase more rapidly with Ra, and data at different $T_{m}$ and $P$ begin to differ from each other. We attribute this phenomenon to NOB effects.

\section{Aspect-ratio dependence}

In Fig. 6 we compare results obtained at a mean temperature $T_{m}=40.00{ }^{\circ} \mathrm{C}$ and pressure $P=51.72$ bar $\left(P / P_{*}\right.$ $=1.062$, Prandtl number $\operatorname{Pr}=2.58)$ in the sample of aspect ratio $\Gamma=0.50$ (open circles) with previously reported results ([46]) for $\Gamma=1.00$ (solid circles). One sees that the NOB

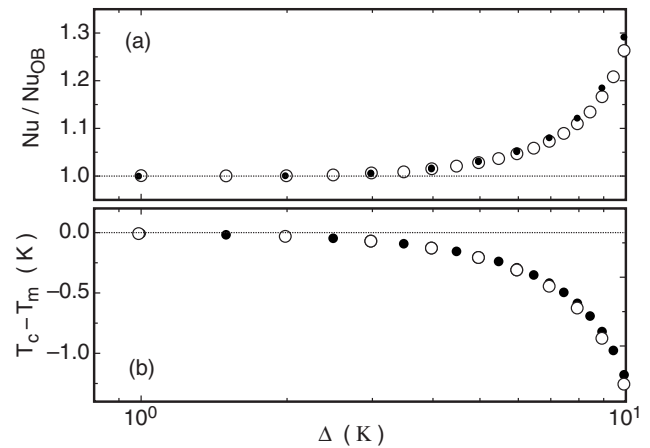

FIG. 6. (a) Ratio of the measured Nusselt number $\mathrm{Nu}$ to the estimate $\mathrm{Nu}_{\mathrm{OB}}$ of the Nusselt number under Boussinesq conditions as a function of the applied temperature difference $\Delta$. (b) Deviation of the center temperature $T_{c}$ from the mean temperature $T_{m}$ as a function of $\Delta$. All measurements were made at $T_{m}=40.00{ }^{\circ} \mathrm{C}$ and a pressure of 51.72 bars $\left(P / P_{*}=1.062\right)$ where the Prandtl number is 2.58. Open symbols, $\Gamma=0.50$. Solid symbols, data from Ref. [46] with $\Gamma=1.00$. 


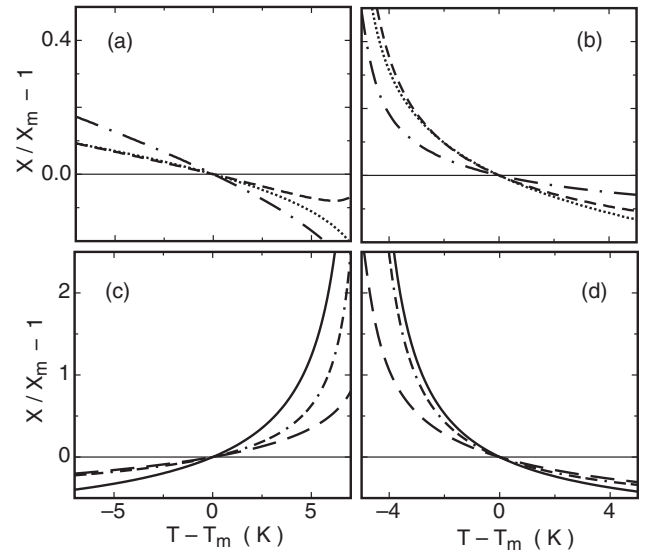

FIG. 7. Ratios $X / X_{m}$ for $P=51.72$ bars $\left(P / P_{*}=1.062\right)$ of several property values $X$ at temperatures $T-T_{m}$ to the value of $X$ at $T_{m}$ (based on Ref. [47]). (a),(c) $T_{m}=27.00{ }^{\circ} \mathrm{C}$. (b), (d) $T_{m}=40.00{ }^{\circ} \mathrm{C}$. (a),(b) Thermal conductivity $\Lambda$ (short-dashed line), density $\rho$ (dotted line), and dynamic viscosity $\eta$ (dash-dotted line). (c),(d) Thermal expansion coefficient $\beta$ (solid line), $\hat{\beta}$ (long-dashed line), and heat capacity at constant pressure $c_{P}$ (double-dash-dotted line). The reference values $X_{m}$ for $27(40){ }^{\circ} \mathrm{C}$ are $\Lambda_{m}=0.07328$ (0.043 43) $\mathrm{W} \mathrm{m} \mathrm{K}^{-1} \mathrm{~K}^{-1}, \quad \rho_{m}=331.12(123.26) \mathrm{kg} \mathrm{m}^{-3}, \quad \eta_{m}=4.030$ (1.502) $\times 10^{-5} \mathrm{~kg} \mathrm{~s}^{-1} \mathrm{~m}^{-1}, \quad \beta_{m}=0.01649(0.03815) \mathrm{K}^{-1}, \quad c_{P, m}=5434$ (7452) $\mathrm{J} \mathrm{kg}^{-1} \mathrm{~K}^{-1}$, and mean Prandtl number $\operatorname{Pr}_{m}=2.99$ (2.58).

effect on $\mathrm{Nu}$ and on the center temperature $T_{c}$ is within our resolution independent of $\Gamma$. This shows, as expected, that the NOB effects are confined essentially to the boundary layers. The length of the sample interior, which is nearly isothermal (see, however, Ref. [59]) regardless of its length, does not have a large influence.

\section{Dependence on fluid properties}

Interesting insight into the influence of various property variations with temperature can be gained by measuring $T_{c}$ and $\mathrm{Nu}$ along an isobar on the two sides of the temperature $T_{\phi}(P)$ at which the critical isochore is reached. In Fig. 7 we show the variation along the isobar $P=51.72$ bars $=1.062 P_{*}$ of the thermal conductivity $\Lambda$, density $\rho$, dynamic viscosity $\eta$, thermal expansion coefficient $\beta$, and heat capacity $c_{P}$ for the cases $T_{m}=27.00{ }^{\circ} \mathrm{C}$ (left panels) and $40.00{ }^{\circ} \mathrm{C}$ (right panels). These two values are on opposite sides of but not quite equidistant from $T_{\phi}=34.97{ }^{\circ} \mathrm{C}$. In the upper two panels one sees that the variations of $\Lambda, \rho$, and $\eta$ are relatively small, have the same trends with $T$ though quantitatively they are somewhat different on the two sides, with maximum changes by less than a factor of 2 over temperature ranges that are small enough to avoid including $T_{\phi}$. On the other hand, the expansion coefficient and the heat capacity (lower two panels) vary by a factor of 5 or more. Thus one might expect them to dominate the NOB effects. Interestingly they have opposite trends with $T-T_{m}$; the temperature derivatives of both $\beta$ and $c_{P}$ are positive below and negative above $T_{\phi}$; that is, below the critical isochore (along the temperature axis), on the more liquidlike side, $\beta$ and $c_{P}$ are smaller at the top (colder) than at the bottom (warmer) end of the sample, with this relationship reversed above the

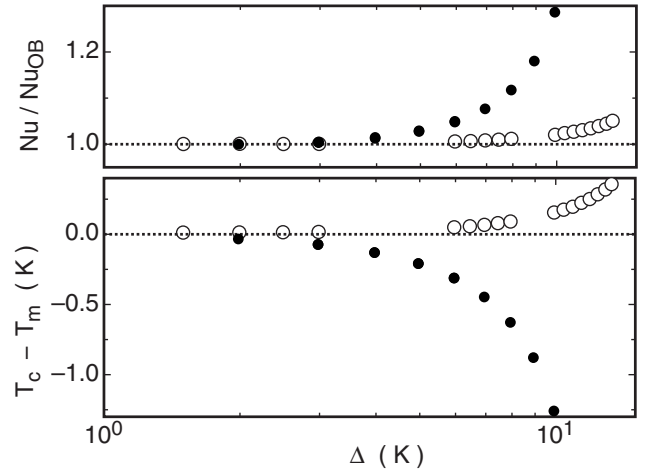

FIG. 8. (a) Ratio of the measured Nusselt number $\mathrm{Nu}$ to the estimate $\mathrm{Nu}_{\mathrm{OB}}$ of the Nusselt number under Boussinesq conditions as a function of the applied temperature difference $\Delta$. (b) Deviation of the center temperature $T_{c}$ from the mean temperature $T_{m}$. These measurements were made for $\Gamma=1.00$ at a pressure of 51.72 bars $\left(P / P_{*}=1.062\right)$. Open symbols, $T_{m}=27.00{ }^{\circ} \mathrm{C} \quad(\operatorname{Pr}=2.99)$. Solid symbols, $T_{m}=40.00{ }^{\circ} \mathrm{C}(\mathrm{Pr}=2.58)$. These two temperatures are on opposite sides of, but not equidistant from, the temperature $T_{\phi}$ $=34.97{ }^{\circ} \mathrm{C}$ where the critical isochore is reached on this isobar.

critical isochore, on the more gaslike side, where $\beta$ and $c_{P}$ decrease from bottom to top.

In Fig. 8 experimental results are presented for $\Gamma=1.00$ at a pressure $P=51.72$ bars $=1.062 P^{*}$. They are for the two mean temperatures $T_{m}=27.00$ (open circles) and $40.00{ }^{\circ} \mathrm{C}$ (solid circles) of Fig. 7 where the Prandtl numbers are 2.99 and 2.58 , respectively. In both cases we used $\Delta$ values sufficiently small so that $T_{t}\left(T_{b}\right)$ only reaches down (up) to $T_{\phi}$, so that the applied temperature difference does not straddle $T_{\phi}$. One sees that the NOB effects increase Nu on both sides of the critical isochore. On the high-temperature side (solid circles). the NOB effect is larger for the same $\Delta$. This is consistent with the larger variation of the fluid properties at equal values of $T-T_{m}$ revealed above in Fig. 7 .

The NOB effect on $T_{c}$ is of opposite sign on the two sides of the critical isochore. For $T<T_{\phi}$ (open circles) NOB conditions increase $T_{c}$ above $T_{m}$, whereas for $T>T_{\phi}$ (solid circles) $T_{c}$ is reduced below $T_{m}$. This observation, in conjunction with the properties shown in Fig. 7, suggests that for these fluids the temperature drops $\Delta_{t, b}$ across the boundary layers are determined primarily by $\beta$ and/or $c_{P}$, with $\Delta_{t}$ $>\Delta_{b}\left(\Delta_{t}<\Delta_{b}\right)$ when $\beta$ and/or $c_{P}$ are smaller (larger) at the cold top end of the sample than at the warm bottom end. As pointed out before [43], for the Nusselt number the contributions to the thermal resistance at the two boundary layers add, and it does not matter much whether the larger or smaller contribution comes from one end or the other. Thus, for $\mathrm{Nu}$ the NOB effect is in the same direction in both cases. As was the case for $\mathrm{Nu}$, the $\mathrm{NOB}$ effect revealed by $T_{c}$ is larger in magnitude above $T_{\phi}$ than it is below. Again we attribute this difference primarily to the difference in the variations of the properties shown in Fig. 7.

In Fig. 9 we show the variation along the isobar $P$ $=55.17$ bars $\left(P / P_{*}=1.132\right)$ of the various properties for the cases $T_{m}=35.00{ }^{\circ} \mathrm{C}$ (left panels) and $41.00{ }^{\circ} \mathrm{C}$ (right panels). These two temperatures are also on opposite sides of and nearly equidistant from the critical isochore, for this pressure 


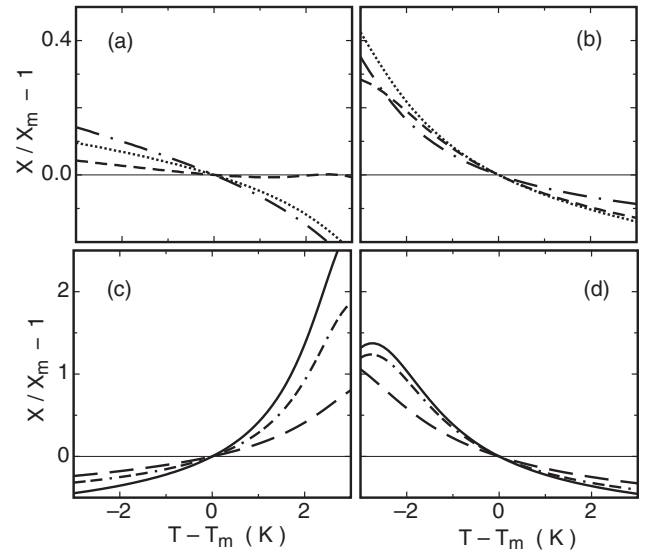

FIG. 9. Ratios $X / X_{m}$ for $P=55.17$ bars $\left(P / P_{*}=1.132\right)$ of several property values $X$ at temperatures $T-T_{m}$ to the value of $X$ at $T_{m}$ (based on Ref. [47]). (a),(c) $T_{m}=35.00{ }^{\circ} \mathrm{C}$. (b),(d) $T_{m}=41.00{ }^{\circ} \mathrm{C}$. (a),(b) Thermal conductivity $\Lambda$ (dashed line), density $\rho$ (dotted line), and dynamic viscosity $\eta$ (dash-dotted line). (c),(d) Thermal expansion coefficient $\beta$ (solid line), $\hat{\beta}$ (long-dashed line), and heat capacity at constant pressure $c_{P}$ (double-dash-dotted line). The reference values $X_{m}$ for $35(41){ }^{\circ} \mathrm{C}$ are $\Lambda_{m}=0.06674$ (0.051 63) $\mathrm{W} \mathrm{m} \mathrm{K}^{-1} \mathrm{~K}^{-1}, \quad \rho_{m}=282.48(153.43) \mathrm{kg} \mathrm{m}^{-3} \mathrm{~K}^{-1}, \quad \eta_{m}=3.168$ $(1.726) \times 10^{-5} \mathrm{~kg} \mathrm{~s}^{-1} \mathrm{~m}^{-1}, \quad \beta_{m}=0.04177(0.06876) \mathrm{K}^{-1}, \quad c_{P, m}$ $=9617(12534) \mathrm{J} \mathrm{kg}^{-1} \mathrm{~K}^{-1}$, and mean Prandtl number $\operatorname{Pr}_{m}$ $=4.56(4.20)$.

at $T_{\phi}=38.06{ }^{\circ} \mathrm{C}$. Again, the variation of the expansion coefficient and the heat capacity (lower two panels) is much larger than that of the other properties. At a given $\left|T-T_{m}\right|$, all the variations are more similar in magnitude on the two sides of $T_{\phi}$ than they were for the case of Fig. 7.

In Fig. 10 experimental results corresponding to the conditions of Fig. 9 are presented for $\Gamma=0.50$. They are for the two mean temperatures $T_{m}=35.00$ (open circles) and $41.00{ }^{\circ} \mathrm{C}$ (solid circles) where the Prandtl numbers are 4.56

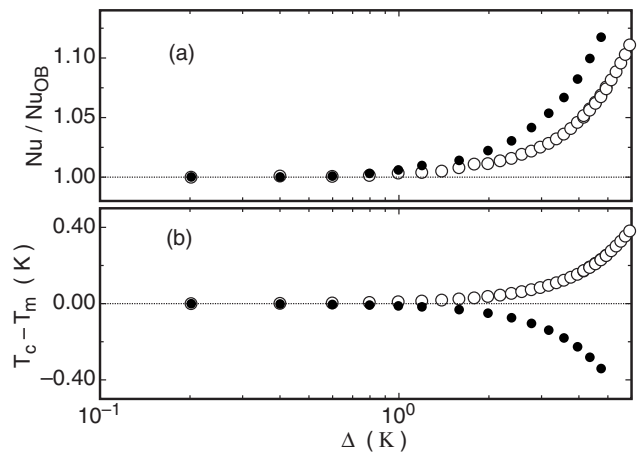

FIG. 10. (a) Ratio of the measured Nusselt number $\mathrm{Nu}$ to the estimate $\mathrm{Nu}_{\mathrm{OB}}$ of the Nusselt number under Boussinesq conditions as a function of the applied temperature difference $\Delta$. (b) Deviation of the center temperature $T_{c}$ from the mean temperature $T_{m}$. These measurements were made for $\Gamma=0.50$ at a pressure of 55.17 bars $\left(P / P_{*}=1.132\right)$. Open symbols: the mean temperature $T_{m}=35{ }^{\circ} \mathrm{C}$ $(\operatorname{Pr}=4.56)$. Solid symbols: $T_{m}=41{ }^{\circ} \mathrm{C}(\operatorname{Pr}=4.20)$. These two temperatures are on opposite sides of and nearly equidistant from the temperature $T_{\phi}=38.06{ }^{\circ} \mathrm{C}$ where the critical isochore is reached on this isobar.

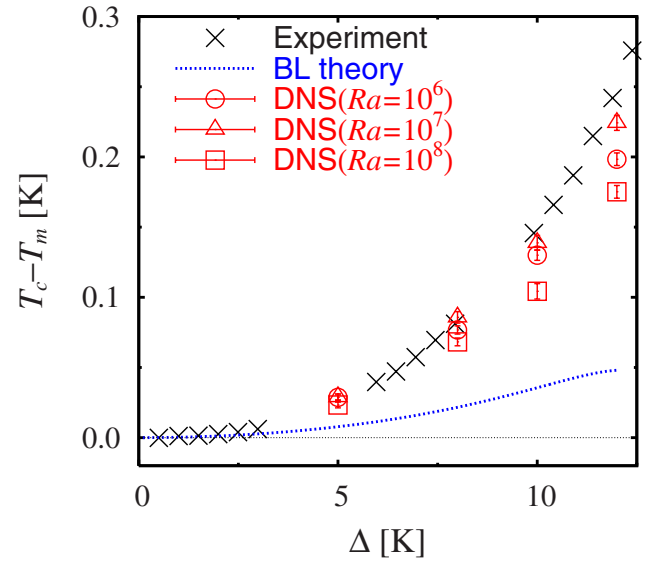

FIG. 11. (Color online) Deviation $T_{c}-T_{m}$ as function of $\Delta$, for $T_{m}=27{ }^{\circ} \mathrm{C}$ and $P / P_{*}=1.062$. The symbols $(X)$ correspond to experimental measurements. The dotted line is obtained from boundary-layer theory. The red symbols $(\bigcirc, \triangle$, and $\square)$ with error bars correspond to the incompressible DNS results described in Sec. $\mathrm{V}$, measured at different $\mathrm{Ra}=10^{6}-10^{8}$. Note that though these Rayleigh numbers are smaller than in the experiments $\left[\mathrm{Ra}=O\left(10^{9}\right)\right.$ $\left.-O\left(10^{10}\right)\right]$, the comparison is still appropriate because the $T_{c}$ shift has proven to be rather independent of Ra for given $\Delta$, provided one is beyond the onset of the chaotic motion at $\mathrm{Ra} \approx 2 \times 10^{5}[45,60]$. For further evidence for the weak Ra dependence of the center temperature we also refer to Table II.

and 4.20 , respectively. In both cases we used $\Delta \leq 6 \mathrm{~K}$ so that $T_{t}\left(T_{b}\right)$ reaches down (up) to $T_{\phi}$ when $T_{m}=41{ }^{\circ} \mathrm{C}\left(35^{\circ} \mathrm{C}\right)$ while the applied temperature difference does not straddle $T_{\phi}$. For this case one sees that the NOB effects on $\mathrm{Nu}$ are similar on the two sides of the critical isochore. Again, the NOB effect on $T_{c}$, although of about the same magnitude, is of opposite sign on the two sides. For $T<T_{\phi}$ (open circles, more liquidlike) NOB conditions increase $T_{c}$ above $T_{m}$, whereas for $T>T_{\phi}$ (solid circles, more gaslike) $T_{c}$ is reduced below $T_{m}$.

\section{COMPARISON BETWEEN BOUNDARY-LAYER THEORY AND THE EXPERIMENTAL RESULTS}

We now compare the experimental measurements from the previous section with the boundary-layer results presented in Sec. II. In particular, since the comparison with experiments at $T_{m}=40{ }^{\circ} \mathrm{C}$ (more gaslike ethane) was already discussed in Ref. [46], finding good agreement between experiment and the extended BL theory, we devote special attention to the measurements at $T_{m}=27{ }^{\circ} \mathrm{C}$ and $P / P_{*}=1.062$ (more liquidlike ethane).

As shown in Fig. 11, the curve for more liquidlike ethane obtained from BL theory considerably deviates from the experimental data. This is remarkably different from the comparison of BL theory with gaseous ethane, presented in [46], where instead a good agreement was observed. This suggests that, even though BL theory reasonably captures NOB effects associated with assumptions OB1 and OB2, further corrections are essential in the present liquidlike ethane case. Among them, deviations from assumption OB3 seem to be 
TABLE I. Expansion coefficients of material properties of ethane around the temperature $T_{m}=27{ }^{\circ} \mathrm{C}$ adapted from [47]. The pressure normalized by its critical value is $P / P_{*}=1.062$. The effective kinematic viscosity, the effective thermal diffusivity, and the buoyancy are written in polynomial form as $\nu(T)$ $\equiv \eta(T) / \rho_{m}=\Sigma_{n=0} A_{n}\left(T-T_{m}\right)^{n}$ (units of $\mathrm{m}^{2} / \mathrm{s}$ ), $\quad \kappa(T) \equiv \Lambda(T) /\left(\rho_{m} c_{p, m}\right)=\Sigma_{n=0} B_{n}\left(T-T_{m}\right)^{n}$ (units of $\mathrm{m}^{2} / \mathrm{s}$ ), and $g\left(1-\rho(T) / \rho_{m}\right)=\Sigma_{n=1} C_{n}\left(T-T_{m}\right)^{n}$ (units of $\mathrm{m} / \mathrm{s}^{2}$ ), respectively. Using the leading coefficient for the buoyancy force, we can write the Rayleigh number as $\mathrm{Ra}=\beta_{m} L^{3} \Delta /\left(\nu_{m} \kappa_{m}\right)$, where $\beta_{m}=C_{1}, \nu_{m}=A_{0}$, and $\kappa_{m}$ $=B_{0}$, which coincides with the usual OB definition. The polynomial expensions for $\beta(T)$ and $\hat{\beta}(T)$ are $g \hat{\beta}(T)=\Sigma_{n=1} C_{n}\left(T-T_{m}\right)^{n-1}$ and $g \beta(T)=\left[\rho_{m} / \rho(T)\right] \Sigma_{n=1} n C_{n}\left(T-T_{m}\right)^{n-1}$.

\begin{tabular}{cccc}
\hline \hline & $\nu$ & $\kappa$ & $g\left(1-\rho / \rho_{m}\right)$ \\
$n$ & $A_{n}$ & $B_{n}$ & $C_{n}$ \\
\hline 0 & $1.21734 \times 10^{-7}\left(\mathrm{~m}^{2} / \mathrm{s}\right)$ & $4.07547 \times 10^{-8}\left(\mathrm{~m}^{2} / \mathrm{s}\right)$ & \\
1 & $-3.38861 \times 10^{-9}\left(\mathrm{~m}^{2} / \mathrm{s} \mathrm{K}\right)$ & $-5.77921 \times 10^{-10}\left(\mathrm{~m}^{2} / \mathrm{s} \mathrm{K}\right)$ & $1.64833 \times 10^{-2}\left(\mathrm{~m}^{2} / \mathrm{s} \mathrm{K}\right)$ \\
2 & $-8.30683 \times 10^{-11}\left(\mathrm{~m}^{2} / \mathrm{s} \mathrm{K}\right)$ & $-7.36395 \times 10^{-12}\left(\mathrm{~m}^{2} / \mathrm{s} \mathrm{K}\right)$ & $6.79967 \times 10^{-4}\left(\mathrm{~m}^{2} / \mathrm{s} \mathrm{K}\right)$ \\
3 & $-5.75280 \times 10^{-12}\left(\mathrm{~m}^{2} / \mathrm{s} \mathrm{K}\right)$ & $-9.06743 \times 10^{-14}\left(\mathrm{~m}^{2} / \mathrm{s} \mathrm{K}\right)$ & $4.53854 \times 10^{-5}\left(\mathrm{~m}^{2} / \mathrm{s} \mathrm{K}\right)$ \\
4 & $-7.64359 \times 10^{-13}\left(\mathrm{~m}^{2} / \mathrm{s} \mathrm{K}\right)$ & $1.49555 \times 10^{-13}\left(\mathrm{~m}^{2} / \mathrm{s} \mathrm{K}\right)$ & $6.13485 \times 10^{-6}\left(\mathrm{~m}^{2} / \mathrm{s} \mathrm{K}\right)$ \\
5 & $-8.70191 \times 10^{-14}\left(\mathrm{~m}^{2} / \mathrm{s} \mathrm{K}\right)$ & $2.56836 \times 10^{-14}\left(\mathrm{~m}^{2} / \mathrm{s} \mathrm{K}\right)$ & $6.94645 \times 10^{-7}\left(\mathrm{~m}^{2} / \mathrm{s} \mathrm{K}\right)$ \\
\hline \hline
\end{tabular}

the natural candidate for the failure of BL theory, since the buoyancy force is not included in the BL equations but apparently affects the thermal convection. Thus, in order to reveal the importance of nonlinear buoyancy in thermal convection, we shall perform direct numerical simulations of the RB problem.

\section{DIRECT NUMERICAL SIMULATIONS}

As shown in Refs. [45,60], two-dimensional direct numerical simulations may be useful for the study of the tiny NOB effects that occur in RB convection in liquids. In particular, even with the restrictions to two-dimensional geometry and to incompressibility of the fluid flow, the effects on the center temperature and the Nusselt-number shift could be reasonably captured in the cases of water and glycerol. However, the liquidlike ethane just above the critical pressure has a stronger temperature dependence of the density than water and glycerol. To quantify this, a comparison between the ethane properties around $T_{m}=27{ }^{\circ} \mathrm{C}$ and $P / P_{*}=1.062$ with water and glycerol around $T_{m}=40{ }^{\circ} \mathrm{C}$ is reported in Fig. 12 . For the case of ethane the incompressible flow approximation seems to be questionable, or at least less justified. But we will show that adopting the same approach used for water and glycerol $[45,60]$ also proves to be useful to study NOB effects in ethane and the results are well consistent with experiment.

Further insight into the problem is given by considering several cases of artificial ethanelike fluids, namely, fluids which have only one, or some, of their material properties dependent on temperature, while the others are kept constant. In particular, as discussed in the previous section, we will examine the relevance of the nonlinear temperature dependence of buoyancy on the center temperature shift $T_{c}-T_{m}$ and take full notice of violation of assumption OB3, which in contrast assumes constant $\partial \rho / \partial T$. We remind the reader that this cannot be taken into account in the extended BL theory presented in Sec. II, while DNSs can well include it.

\section{A. Numerical simulation approach}

To handle the numerical effort we restrict ourselves to incompressible and even two dimensional flow. The equations governing non-Oberbeck-Boussinesq convection in incompressible fluid flow are the incompressibility condition

$$
\partial_{i} u_{i}=0
$$

the Navier-Stokes equation

$$
\begin{aligned}
\rho_{m}\left(\partial_{t} u_{i}+u_{j} \partial_{j} u_{i}\right)= & -\partial_{i} p+\partial_{j}\left[\eta\left(\partial_{j} u_{i}+\partial_{i} u_{j}\right)\right] \\
& +g \rho_{m}\left(1-\rho / \rho_{m}\right) \delta_{i 3},
\end{aligned}
$$

and the heat-transfer equation

$$
\rho_{m} c_{p, m}\left(\partial_{t} T+u_{j} \partial_{j} T\right)=\partial_{j}\left(\Lambda \partial_{j} T\right) .
$$

Here, $\delta_{i 3}$ is the Kronecker symbol. The density is assumed to be constant and its value $\rho_{m}$ is fixed at that of the temperature $T_{m}$, except in the buoyancy term, where the full nonlinear temperature dependence of $\rho(T)$ is implemented. The dynamic viscosity $\eta(T)$ and the heat conductivity $\Lambda(T)$ are also both temperature and thus space dependent. The isobaric specific heat capacity $c_{P}$ is assumed to be constant, its value being $c_{p, m}$ (in contrast to real ethane). The experimentally known temperature dependences of $\rho, \eta$, and $\Lambda$ and the values of the parameters $\rho_{m}$ and $c_{p, m}$ for ethane are given in [47] and, for better reference, are reported in Table I in the specific form implemented in our DNSs.

For consistency with the experimental measurements and with the BL theoretical analyses presented above for liquidlike ethane, we chose the arithmetic mean temperature to be $T_{m}=27{ }^{\circ} \mathrm{C}$ and the pressure as $P / P_{*}=1.062$.

\section{B. Numerical results: $T_{c}$ shift in liquidlike ethane}

From Fig. 11 we can conclude that the DNS captures the experimental measurements of the center temperature shift $T_{c}-T_{m}$ as a function of $\Delta$ quite reasonably. The quality of the agreement with the available ethane data is similar to the one we have observed for glycerol [45] and for water [60]. This 

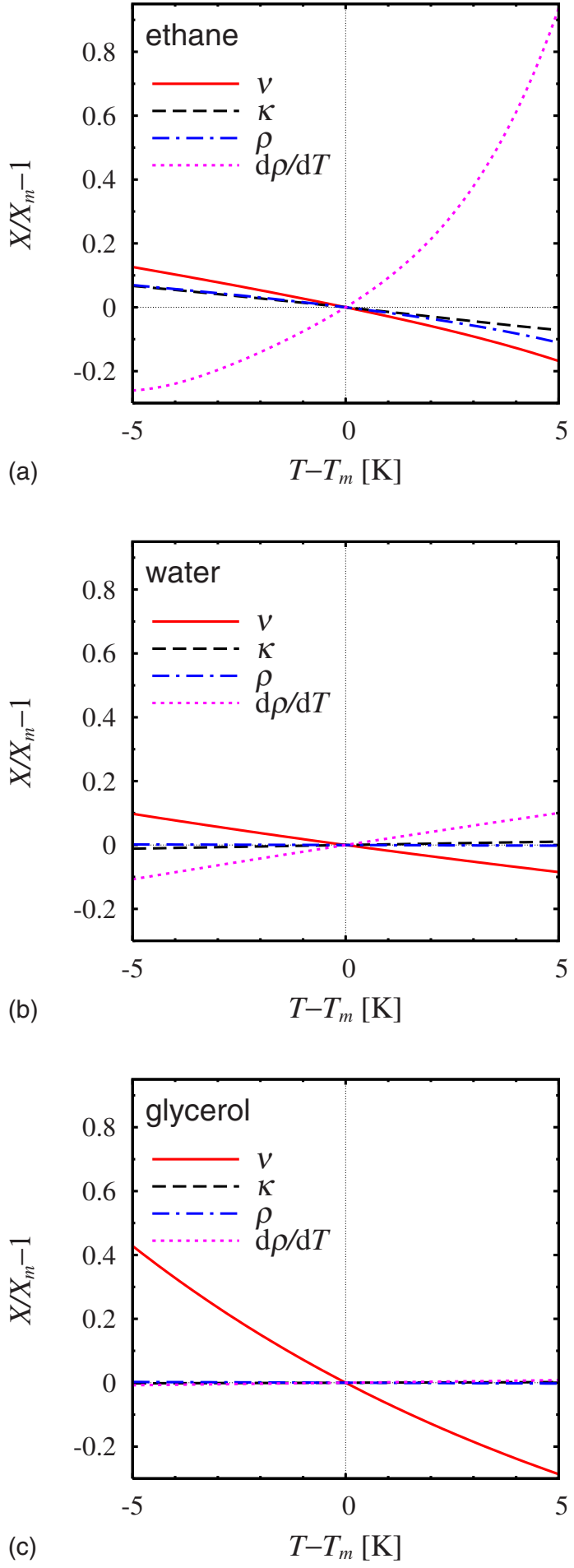

FIG. 12. (Color online) Temperature dependences of the material properties $\nu, \kappa$, and $\rho$ in the relevant $T$ range. $d \rho / d T$ is also displayed. (a) Ethane around the temperature $T_{m}=27{ }^{\circ} \mathrm{C}$ adapted from [47]. The pressure is $P / P_{*}=1.062$. (b),(c) Fuid properties for water (b) and glycerol (c), respectively, around the temperature $T_{m}=40{ }^{\circ} \mathrm{C}$ (which has been studied in [45] and [60]). Note the significant variation of the density $\rho$ with $T$ in the case of ethane, as compared to the two liquids. In ethane, $\rho / \rho_{m}-1$ varies from about 0.07 for $T-T_{m}=-5 \mathrm{~K}$ to about -0.1 for $T-T_{m}=+5 \mathrm{~K}$, whereas in glycerol $\rho_{m}$ is practically independent of $T$. The strong variation of $\rho$ for ethane follows from the large $d \rho / d T$ (also shown). also serves as a further validation of our numerical approach.

We note that for water and for glycerol the $T_{c}$ shift obtained by the extended BL theory [43] is nearly the same as calculated by DNSs (see $[45,60]$ ). In contrast, for the liquidlike ethane, the extended and even compressible BL theory provides only the right trend in the shift, but cannot capture its amplitude (see again Fig. 11). This observation supports our guess about the relevance of the nonlinear $T$ dependence of $\rho(T)$ and thus of buoyancy. This latter is fully included in the DNSs, as described in the previous section, while in BL theory it cannot be taken care of.

One of the advantages of the DNSs as compared to real experiments is that the material properties are easily and independently tunable. Therefore, the dynamics of hypothetical ethanelike liquids can also be addressed. In the next subsection we shall see how this approach is useful in understanding the effects of the temperature dependence of the various material properties on the center temperature shift.

\section{C. $T_{c}$ shift in hypothetical fluids}

To obtain more insight into the physical origin of the nonOberbeck-Boussinesq $T_{c}$ shift, we consider NOB corrections for hypothetical ethanelike fluids in which at least one of the temperature dependences of $\kappa(T), \nu(T)$, and $\hat{\beta}(T)$ is switched off, and fixed at the OB values $\kappa_{m}, \nu_{m}$, and $\beta_{m}$. The quantity $\hat{\beta}(T)$, defined in Eq. (2), is useful for the classification of the hypothetical fluids discussed in the following sections, but $\hat{\beta}(T)$ is not explicitly introduced into the DNSs, in which the density difference $\rho(T)-\rho_{m}$ is taken instead; see Eq. (15) and Table I. Finally, we recall that $\hat{\beta}(T)=\beta_{m}$, the usual thermal expansion coefficient, if the fluid density is a linear function of the temperature around $T_{m}$ (i.e., if the condition OB3 holds). In that case the thermal expansion coefficient $\beta(T)$ $=\beta_{m} /\left[1-\left(T-T_{m}\right) \beta_{m}\right]$ still depends on $T$ unless $\beta \Delta \ll 1$.

For convenience hereafter we will call the two classes of artificial fluids, based, respectively, on the full nonlinear NOB buoyancy force and on the linear OB approximation as defined by approximation OB3, as the $\hat{\beta}(T)$ fluids and $\beta_{m}$ fluids.

In Fig. 13 we present the DNS results of the normalized temperature shift $\left(T_{c}-T_{m}\right) / \Delta$ for several types of hypothetical fluids. The numeral values are given in Table II. One clearly observes in the figures and in the table that the temperature dependence of the thermal expansion function $\hat{\beta}(T)$ apparently is relevant for the shift of $T_{c}$. What can also be noticed in particular from Table II is that the corrections of the center temperature originating from the temperature dependence of either $\nu, \kappa$, or $\beta$ are approximately additive (i.e., add "linearly"): for example, the center temperature corrections of the fluids with $\left[\left(\beta_{m}, \kappa_{m}, \nu(T)\right]\right.$ and with $\left[\beta_{m}, \kappa(T), \nu_{m}\right]$ add to that of the fluid with $\left[\beta_{m}, \kappa(T), \nu(T)\right]$, etc. Note that this additivity is in contrast to what had been found within the extended BL theory of Ref. [45], where the full compressibility of the density was taken into consideration. Instead, in DNSs we have restricted attention to incompressible flow. 

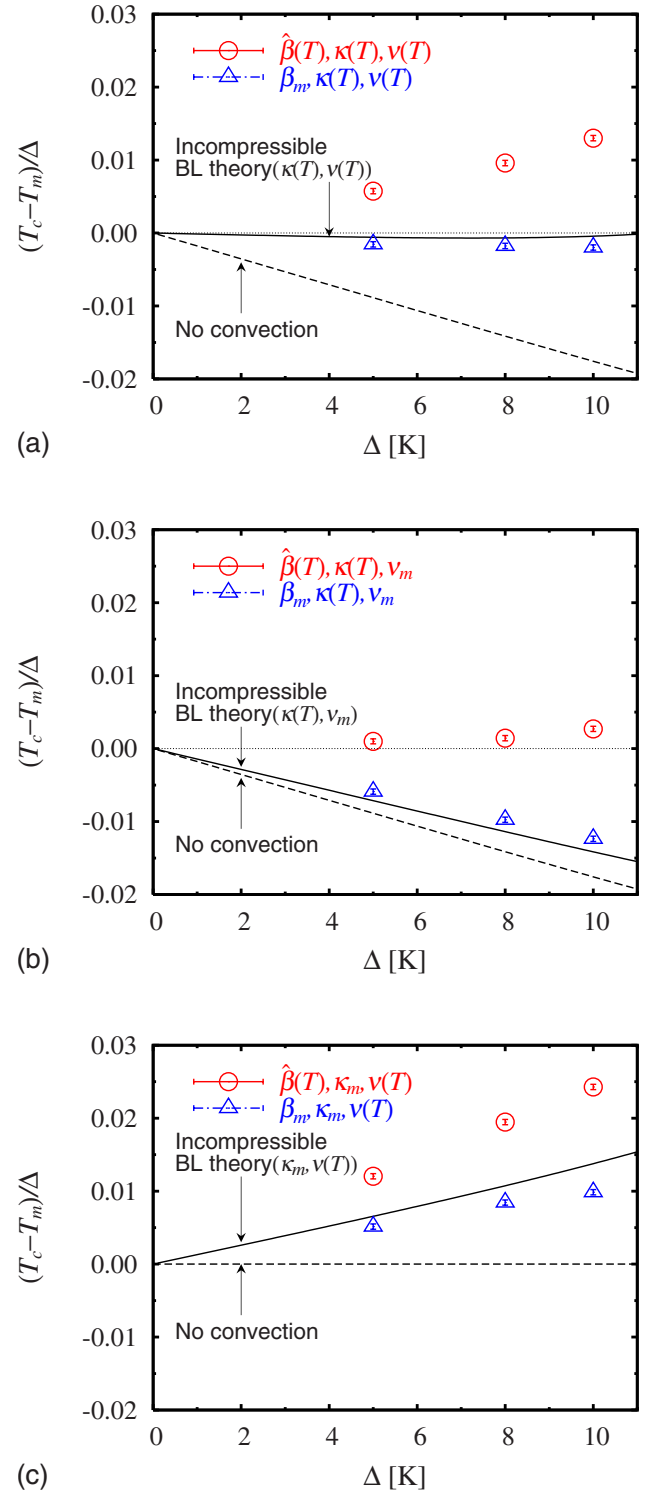

FIG. 13. (Color online) Normalized center temperature shift $\left(T_{c}-T_{m}\right) / \Delta$ versus the temperature difference $\Delta$ for several hypothetical liquids. We consider the following six hypothetical liquids: $(\hat{\beta}(T), \kappa(T), \nu(T)), \quad\left(\beta_{m}, \kappa(T), \nu(T)\right), \quad\left(\hat{\beta}(T), \kappa(T), \nu_{m}\right), \quad\left(\beta_{m}, \kappa(T)\right.$, $\left.\nu_{m}\right),\left(\hat{\beta}(T), \kappa_{m}, \nu(T)\right)$, and $\left(\beta_{m}, \kappa_{m}, \nu(T)\right)$. In particular, in each panel we compare two cases, which differ only by their buoyancy's $T$ dependence, i.e., $\hat{\beta}(T)$ instead of $\beta_{m}$. The symbols indicate the simulation results at Rayleigh number $\mathrm{Ra}=10^{6}$. The circles $(\bigcirc)$ correspond to the cases in which the full temperature dependence of the buoyancy $\left(T-T_{m}\right) \hat{\beta}(T)$ is taken into account, while the triangles $(\triangle)$ represent the cases where only the linear temperature dependence $\left(T-T_{m}\right) \beta_{m}$ is considered. The solid line shows the prediction of boundary-layer theory with an incompressible flow assumption [43]. The dashed line stems from the solution with no convective flow $\partial_{z}\left[\kappa(T) \partial_{z} T\right]=0$ with boundary conditions $T=T_{b}$ at $z=0$ and $T$ $=T_{t}$ at $z=L$. See also Table II.

\section{1. $\hat{\beta}(T)$ and $\beta_{m}$ fluids and extended BL theory}

In Fig. 13 we also compare fluids of $\hat{\beta}(T)$ and $\beta_{m}$ types with equal properties of thermal conductivity and kinematic viscosity on the different panels. For convenient comparison the predictions by the extended BL theory under the assumption of fluid incompressibility [43] are also shown for the hypothetical fluids. Note that in this case the chosen type of buoyancy force need not be specified, because BL theory does not capture it: For the BL theory $\hat{\beta}(T)$ and $\beta_{m}$ ethane are not distinguishable with respect to buoyancy. As a reference, the $T_{c}$-shift value in the purely conductive case $\left(u_{i}=0\right.$ everywhere) is also reported. The $T_{c}$ shift in this case is not zero for fluids with $\kappa=\kappa(T)$ as a result of the solution of the heat conduction equation $\kappa \partial_{z}^{2} T+\frac{d \kappa}{d T}\left(\partial_{z} T\right)^{2}=0$ with $\left.\frac{\partial \kappa}{\partial T}\right|_{P} \neq 0$.

We now discuss our main findings. The change in $T_{c}$ for the hypothetical liquids with $\beta_{m}$ relative to the ones with $\hat{\beta}(T)$ is comparable for given $\Delta$. More importantly, we find that the deviation $\left(T_{c}-T_{m}\right) / \Delta$ calculated with DNS is well captured by the BL theory for the cases of artificial $\beta_{m}$ fluids, i.e., for fluids where $\rho(T)$ is assumed to be a linear function of $T$. In contrast, BL theory is always far from the $T_{c}$ shift obtained for the more real $\hat{\beta}(T)$ ethane. This indicates that the extended BL theory captures the NOB effect well once the assumption OB3 is satisfied, even if assumptions OB1 and OB2 are violated, but it does not correctly describe the NOB effects if assumption OB3 is violated. Furthermore, the DNS results reveal that the $T_{c}$ shift is always enhanced if a temperature dependence of the thermal expansion function $\hat{\beta}(T)$ determines the buoyancy, i.e., if $\rho(T)$ depends nonlinearly on $T$.

\section{Mirror transformation $\hat{\beta}(T) \rightarrow \hat{\beta}\left(2 T_{m}-T\right)$}

To quantitatively appreciate the effect of the temperature dependence in each material property individually also at different Rayleigh numbers, $\mathrm{Ra}=10^{6}-10^{8}$, we list the $T_{c}$ shifts for several hypothetical ethanelike fluids in Table II. Since here our primary concern is the influence of the thermal expansion function, in addition to the $\hat{\beta}(T)$ and $\beta_{m}$ fluids a new class of hypothetical fluids is introduced. We consider $\hat{\beta}\left(2 T_{m}-T\right)$ fluids obtained by the mirror transformation $\hat{\beta}(T) \rightarrow \hat{\beta}\left(2 T_{m}-T\right)$. As schematically shown in Fig. 14, this transformation reverses the nonlinearity in the buoyancy force with respect to $T-T_{m}$. The comparison between the cases $\left(\hat{\beta}(T), \kappa_{m}, \nu_{m}\right)$ and $\left(\hat{\beta}\left(2 T_{m}-T\right), \kappa_{m}, \nu_{m}\right)$-cases 4 and 8 in Table II shows that the effect of the mirror transformation on the output parameter $T_{c}$ is to change the sign of $\left(T_{c}\right.$ $\left.-T_{m}\right) / \Delta$ while preserving its modulus. Furthermore, the deviation of $\left(T_{c}-T_{m}\right) / \Delta$ for all $\beta_{m}$ fluids (cases 9-11) relative to the $\hat{\beta}(T)$ fluids (cases 1-3) is always positive, while it is always negative relative to the $\hat{\beta}\left(2 T_{m}-T\right)$ fluids (cases 5-7). These features hold at all the studied Ra numbers. Therefore, we conclude that the shift $T_{c}-T_{m}$ is sensitive to the sign of the slope of $\hat{\beta}(T)$ or, equivalently, to the sign of the nonlinear term in the buoyancy factor $1-\rho(T) / \rho_{m}$. More precisely speaking, the mirror transformation changes the signs of the even-order coefficients $C_{2}$ and $C_{4}$ defined in Table I. Obviously $C_{2}$ is the larger and thus the more relevant coefficient. These features are absent in water and in glycerol, because for those the temperature dependence of $\rho(T)$ is much less pronounced. 
TABLE II. DNS results for the center temperature shift $T_{c}-T_{m}$ normalized by the temperature difference $\Delta=10 \mathrm{~K}$ for several hypothetical fluids. The effective thermal expansion function is given by $\hat{\beta}(T)$ $=g^{-1} \sum_{n=1} C_{n}\left(T-T_{m}\right)^{n-1}$ (units of $1 / \mathrm{K}$ ). Using the expansion coefficients $C_{n}$ listed in Table I, we write the buoyancy for the case of $\hat{\beta}\left(2 T-T_{m}\right)$ as $g\left(1-\rho(T) / \rho_{m}\right)=\Sigma_{n=1}(-1)^{n+1} C_{n}\left(T-T_{m}\right)^{n}$ (units of $\mathrm{m} / \mathrm{s}^{2}$ ), and in the $\beta_{m}$ case as $g\left(1-\rho(T) / \rho_{m}\right)=C_{1}\left(T-T_{m}\right)$ (units of $\mathrm{m} / \mathrm{s}^{2}$ ). Although the center temperature shift for the OB case (case 12) should be essentially zero due to the top-bottom symmetry, the mean value determined from the DNS result is nonzero since the sampling time for taking the statistics is finite. Note that measurements of the temperature shift in the $\mathrm{OB}$ case are all, within statistical uncertainty, compatible with zero.

\begin{tabular}{|c|c|c|c|c|c|c|}
\hline Case & $\hat{\beta}$ & $\kappa$ & $\nu$ & $\begin{array}{c}100\left(T_{c}-T_{m}\right) / \Delta \\
\text { at } \mathrm{Ra}=10^{6}\end{array}$ & $\begin{array}{c}100\left(T_{c}-T_{m}\right) / \Delta \\
\text { at } \mathrm{Ra}=10^{7}\end{array}$ & $\begin{array}{c}100\left(T_{c}-T_{m}\right) / \Delta \\
\text { at } \mathrm{Ra}=10^{8}\end{array}$ \\
\hline 1 (NOB) & $\hat{\beta}(T)$ & $\kappa(T)$ & $\nu(T)$ & $1.3003 \pm 0.0369$ & $1.3879 \pm 0.0527$ & $1.0788 \pm 0.0362$ \\
\hline 2 & $\hat{\beta}(T)$ & $\kappa(T)$ & $\nu_{m}$ & $0.2699 \pm 0.0356$ & $0.2174 \pm 0.0515$ & $0.1251 \pm 0.0414$ \\
\hline 3 & $\hat{\beta}(T)$ & $\kappa_{m}$ & $\nu(T)$ & $2.4283 \pm 0.0361$ & $2.4351 \pm 0.0496$ & $2.4370 \pm 0.0462$ \\
\hline 4 & $\hat{\beta}(T)$ & $\kappa_{m}$ & $\nu_{m}$ & $1.4805 \pm 0.0355$ & $1.4320 \pm 0.0735$ & $1.3796 \pm 0.0534$ \\
\hline 5 & $\hat{\beta}\left(2 T_{m}-T\right)$ & $\kappa(T)$ & $\nu(T)$ & $-1.5953 \pm 0.0361$ & $-1.7946 \pm 0.0506$ & $-1.5868 \pm 0.0320$ \\
\hline 6 & $\hat{\beta}\left(2 T_{m}-T\right)$ & $\kappa(T)$ & $\nu_{m}$ & $-2.6382 \pm 0.0357$ & $-2.7458 \pm 0.0496$ & $-2.5516 \pm 0.0496$ \\
\hline 7 & $\hat{\beta}\left(2 T_{m}-T\right)$ & $\kappa_{m}$ & $\nu(T)$ & $-0.4738 \pm 0.0375$ & $-0.4578 \pm 0.0580$ & $-0.3358 \pm 0.0354$ \\
\hline 8 & $\hat{\beta}\left(2 T_{m}-T\right)$ & $\kappa_{m}$ & $\nu_{m}$ & $-1.4878 \pm 0.0366$ & $-1.5358 \pm 0.0504$ & $-1.4013 \pm 0.0363$ \\
\hline 9 & $\beta_{m}$ & $\kappa(T)$ & $\nu(T)$ & $-0.1983 \pm 0.0361$ & $-0.2043 \pm 0.0467$ & $-0.2691 \pm 0.0587$ \\
\hline 10 & $\beta_{m}$ & $\kappa(T)$ & $\nu_{m}$ & $-1.2369 \pm 0.0363$ & $-1.1883 \pm 0.0611$ & $-1.1851 \pm 0.0369$ \\
\hline 11 & $\beta_{m}$ & $\kappa_{m}$ & $\nu(T)$ & $0.9852 \pm 0.0364$ & $1.0834 \pm 0.0576$ & $0.9916 \pm 0.0259$ \\
\hline $12(\mathrm{OB})$ & $\beta_{m}$ & $\kappa_{m}$ & $\nu_{m}$ & $0.0171 \pm 0.0381$ & $-0.0484 \pm 0.0548$ & $0.0271 \pm 0.0387$ \\
\hline
\end{tabular}

\section{Test of the linearity of the $T_{c}-T_{m}$ shift}

Looking at Table II in more detail, we find that all changes in $\left(T_{c}-T_{m}\right) / \Delta$, that stem from the nonlinear $T$ dependence of the buoyancy force, i.e., from the differences between $\beta_{m}$ and $\hat{\beta}(T)$ [or $\left.\hat{\beta}\left(2 T_{m}-T\right)\right]$, but have the same temperature dependences of $\kappa$ and $\nu$, are comparable. To emphasize this feature, we look at the differences $T_{c} \mid \hat{\beta}(T)$ - $\left.T_{c}\right|_{\beta_{m}}\left[\right.$ or $\left.-\left(T_{c}\left|\hat{\beta}\left(2 T_{m}-T\right)-T_{c}\right|_{\beta_{m}}\right)\right]$ and plot them versus $\Delta$ as well as versus Ra; see Fig. 15. We find a good collapse of the data onto a single curve for various temperature dependences of $\kappa$ and $\nu$. In particular, the comparison between the middle and bottom panels of Fig. 15 leads to the relation $T_{c} \mid \hat{\beta}(T)$ $-\left.T_{c}\right|_{\beta_{m}}=-\left(T_{c}\left|\hat{\beta}\left(2 T_{m}-T\right)-T_{c}\right|_{\beta_{m}}\right)$, which indicates that the $T_{c}$ change is dominated by the quadratic term in $1-\rho(T) / \rho_{m}$, but is almost independent of $\kappa$ and $\nu$. This observation may

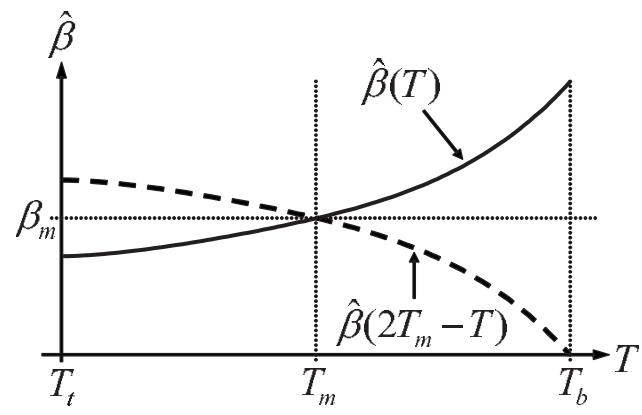

FIG. 14. Schematic plot of the mirror transformation $\hat{\beta}(T)$ $\rightarrow \hat{\beta}\left(2 T_{m}-T\right)$ of the thermal expansion coefficient $\hat{\beta}$. be important for further attempts to improve the extended NOB BL theory.

\section{CONCLUSIONS}

In this paper we first presented in full detail the extension of boundary-layer theory to the case of compressible NOB fluids in a Rayleigh-Bénard system. The theory predicts a deviation of the center temperature $T_{c}$ from the arithmetic mean temperature $T_{m}$ between the top and bottom plates, i.e., $T_{c}-T_{m} \neq 0$.

Second, the theory has been tested against experimental data for ethane near the critical point in its more liquidlike phase. Data come from a series of experiments in cylindrical cells of aspect ratio $\Gamma=0.5$ and 1 , reaching $\mathrm{Ra}$ numbers $O\left(10^{10}\right)$. The experimental measurements at $T_{m}=27{ }^{\circ} \mathrm{C}$, $P / P_{*}=1.062$, and $\Gamma=1$ have been chosen for comparison with those at $T_{m}=40{ }^{\circ} \mathrm{C}$. Contrary to the good agreement observed for the case of gaslike ethane [43], the BL theory here gives much smaller values of the center temperature shift than experiment.

Third, direct numerical simulations, based on $T$-dependent material parameters but still within the incompressible approximation and a two-dimensional domain, have been performed to get more insight into the observed discrepancy between experiment and extended BL theory. The DNS results provide a satisfactory agreement with experiment both in the gaslike and in the liquidlike cases. Several hypothetical ethanelike fluids have been investigated too. Our analysis shows that the extended BL predictions fail 

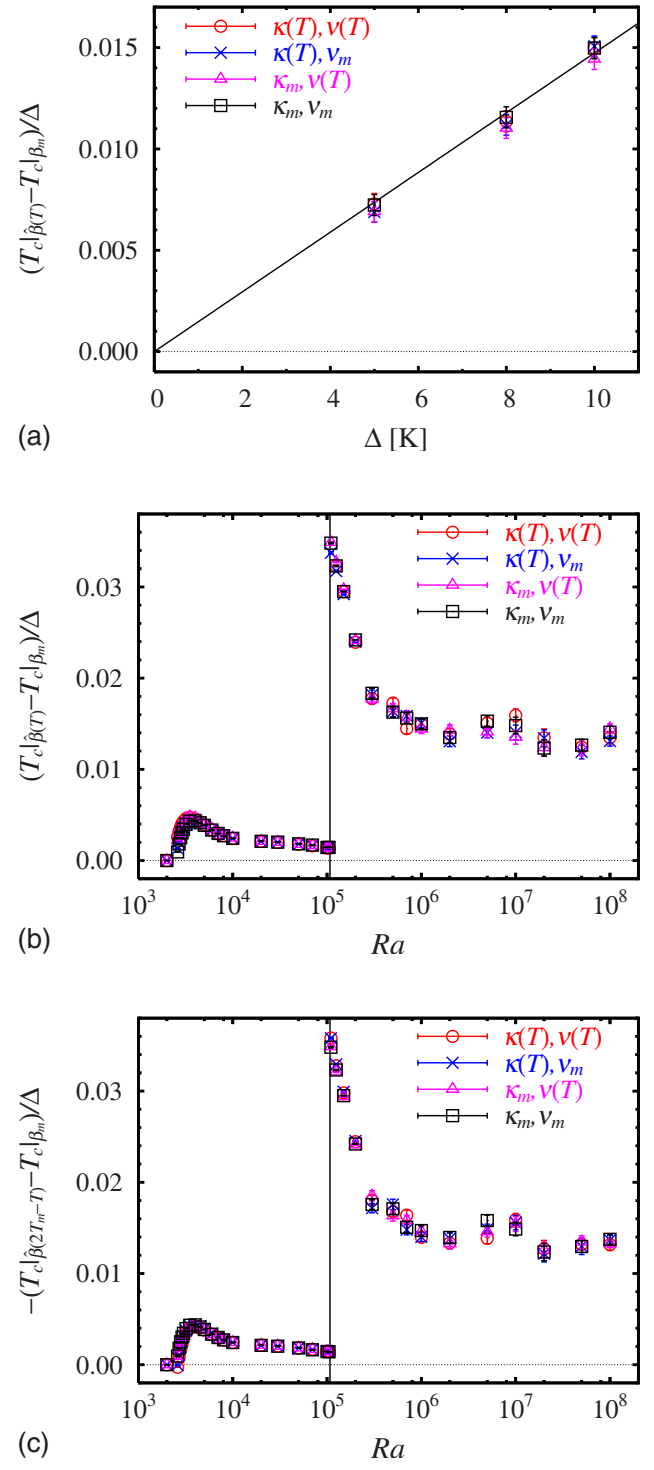

FIG. 15. (Color online) Effect of the temperature dependence of the thermal expansion coefficient $\hat{\beta}$ on the shift of the center temperature for several hypothetical fluids. DNS results for the normalized temperature difference $\left(T_{c}\left|\hat{\beta}_{(T)}-T_{c}\right|_{\beta_{m}}\right) / \Delta$ [or $-\left(T_{c} \mid \hat{\beta}_{\left(2 T_{m}-T\right)}\right.$ $\left.\left.-\left.T_{c}\right|_{\beta_{m}}\right) / \Delta\right]$ with the same temperature dependence of the thermal diffusivity $\kappa$ and the kinematic viscosity $\nu$ are plotted; here $T_{c} \mid \hat{\beta}(T)$ denotes the center temperature with the full temperature dependence of the buoyancy $g\left(1-\rho / \rho_{m}\right)$ as given in Table I, and $\left.T_{c}\right|_{\beta_{m}}$ denotes that with the linear temperature dependence $g(1$ $\left.-\rho(T) / \rho_{m}\right)=C_{1}\left(T-T_{m}\right) \quad$ only. The top panel shows $\left(T_{c} \mid \hat{\beta}(T)\right.$ $\left.-\left.T_{c}\right|_{\beta_{m}}\right) / \Delta$ versus $\Delta$ at fixed Rayleigh number $\mathrm{Ra}=10^{6}$. The solid line shows the linear fit $1.473 \times 10^{-3} \mathrm{~K}^{-1} \times \Delta$ for the case $\hat{\beta}(T), \kappa_{m}, \nu_{m}$ (see Table II). The middle panel shows $\left(T_{c} \mid \hat{\beta}(T)\right.$ $\left.-\left.T_{c}\right|_{\beta_{m}}\right) / \Delta$ versus Ra at fixed temperature difference $\Delta=10 \mathrm{~K}$. The bottom panel has the same parameters as the middle one, except it shows $-\left(T_{c}\left|\hat{\beta}\left(2 T_{m}-T\right)-T_{c}\right|_{\beta_{m}}\right) / \Delta$ for the case of mirror-transformed $\hat{\beta}$.

whenever the nonlinear temperature dependence of the density $\rho(T)$ is implemented in the numerical simulations. Furthermore, if the dependence of $\hat{\beta}(T)$ on $T$ dominates the NOB effects, the sign of the linear term in the effective ex- pansion function $\hat{\beta}(T)$ is responsible for the sign of the variations of $T_{c}$ as compared to the OB value $T_{c}=T_{m}$. But even if the difference $\hat{\beta}(T)-\beta_{m}$ is a nonlinear function of the temperature, the tiny $T_{c}$ shift detected in our simulations is proportional to $T_{c}\left|\hat{\beta}(T)-T_{c}\right|_{\beta_{m}}$.

Our results can be summarized in the following physical picture of the surprising phenomenon that the center temperature $T_{c}$ in liquidlike ethane near its critical point increases and the center becomes warmer than the arithmeric mean temperature $T_{m}$, while in gaslike ethane the center gets colder and $T_{c}$ is less than $T_{m}$. Namely, in the liquidlike case the buoyancy proportional to $\left(T-T_{m}\right) \hat{\beta}(T)$ is larger at the bottom and smaller at the top, supporting the uprising warmer plumes more than the down-coming colder top plumes. This brings predominantly hotter material into the bulk. For gaslike ethane the buoyancy is larger at the cooler top, which accelerates the downgoing cold plumes preferentially over the uprising warm plumes from the bottom, which experience a weaker buoyancy. This in turn brings more cooler material into the bulk, leading to $T_{c}<T_{m}$. It is the sign of the slope of $\hat{\beta}$ (or of $\beta$ ), that is the relevant quantity.

The more general lesson that can be drawn from this paper is that there is a plethora of origins of NOB corrections. Which one dominates can be determined only by taking a detailed look at the temperature dependence of all material parameters. Both the extended BL theory and 2D DNSs are useful tools to judge which temperature dependence is the most relevant one or whether they all matter, as we now have often seen.

\section{ACKNOWLEDGMENTS}

This work is part of the research program of the Stichting voor Fundamenteel Onderzoek der Materie (FOM), which is financially supported by the Nederlandse Organisatie voor Wetenschappelijk Onderzoek (NWO). The experimental work was supported by U.S. National Science Foundation Grant No. DMR07-02111.

\section{APPENDIX A: BOUNDARY-LAYER EQUATIONS}

\section{Viscous boundary layer}

Consider two-dimensional flow over a flat plate, such that the main wind velocity $U$ does not depend on $x$ and $\lim _{z \rightarrow \infty} u_{x}(x, z)=U$. Then, the $x$ momentum near the wall is governed by [50]

$$
\begin{aligned}
\rho\left\{u_{x} \frac{\partial u_{x}}{\partial x}+u_{z} \frac{\partial u_{x}}{\partial z}\right\}= & \eta\left\{\frac{\partial^{2} u_{x}}{\partial x^{2}}+\frac{\partial^{2} u_{x}}{\partial z^{2}}\right\}+\frac{\partial \eta}{\partial z}\left\{\frac{\partial u_{x}}{\partial z}+\frac{\partial u_{z}}{\partial x}\right\} \\
& +\frac{\partial}{\partial x}\left\{\left(\frac{\eta}{3}+\zeta\right)\left(\frac{\partial u_{x}}{\partial x}+\frac{\partial u_{z}}{\partial z}\right)\right\},
\end{aligned}
$$

where $\eta$ is the dynamic shear viscosity and $\zeta$ the volume expansion viscosity.

To appraise the dominant structure of Eq. (A1) at sufficiently large Reynolds numbers, we follow Prandtl's scaling:

$$
x=L \tilde{x}
$$




$$
\begin{gathered}
z=\frac{L}{\sqrt{\operatorname{Re}}} \tilde{z}, \\
u_{x}=U \tilde{u}_{x}, \\
u_{z}=\frac{U}{\sqrt{\operatorname{Re}}} \tilde{u}_{z},
\end{gathered}
$$

where $L$ denotes the typical length of the plate in the flow direction and $\operatorname{Re}=L U \rho_{m} / \eta_{m}$ the Reynolds number. The index $m$ indicates that the fluid properties are evaluated at a thermodynamic reference state $\left(T_{m}, P_{m}, \rho_{m}\right)$, which is adopted in the nondimensionalization of $\rho \equiv \rho_{m} \widetilde{\rho}$ and $\eta$ $\equiv \eta_{m} \tilde{\eta}$. Then, substituting (A2)-(A5) into (A1), one finds

$$
\begin{aligned}
\widetilde{\rho}\left\{\tilde{u}_{x} \frac{\partial \tilde{u}_{x}}{\partial \tilde{x}}+\tilde{u}_{z} \frac{\partial \tilde{u}_{x}}{\partial \widetilde{z}}\right\}= & \tilde{\eta}\left\{\frac{1}{\operatorname{Re}} \frac{\partial^{2} \tilde{u}_{x}}{\partial \tilde{x}^{2}}+\frac{\partial^{2} \tilde{u}_{x}}{\partial \widetilde{z}^{2}}\right\} \\
& +\frac{\partial \tilde{\eta}}{\partial \widetilde{z}}\left\{\frac{\partial \tilde{u}_{x}}{\partial \widetilde{z}}+\frac{1}{\operatorname{Re}} \frac{\partial \tilde{u}_{z}}{\partial \tilde{x}}\right\} \\
& +\frac{1}{\operatorname{Re}} \frac{\partial}{\partial \tilde{x}}\left\{\tilde{\eta}_{0}\left(\frac{\partial \tilde{u}_{x}}{\partial \tilde{x}}+\frac{\partial \tilde{u}_{z}}{\partial \widetilde{z}}\right)\right\},
\end{aligned}
$$

where $\widetilde{\eta}_{0}=\tilde{\eta}\left(\frac{1}{3}+\frac{\xi}{\eta}\right)$. Clearly, all terms on the left-hand side of Eq. (A6) are of order unity. However, this is not the case on the right-hand side of (A6): only the viscous contributions involving transverse gradients of $u_{x}$ are of order 1 ; the remaining terms are of order $1 / \mathrm{Re}^{1}$

Therefore, at large $\mathrm{Re}$, the dominant part of the $x$-momentum equation is given by

$$
\widetilde{\rho}\left\{\tilde{u}_{x} \frac{\partial \tilde{u}_{x}}{\partial \widetilde{x}}+\tilde{u}_{z} \frac{\partial \tilde{u}_{x}}{\partial \widetilde{z}}\right\}=\tilde{\eta} \frac{\partial^{2} \tilde{u}_{x}}{\partial \widetilde{z}^{2}}+\frac{\partial \widetilde{\eta}}{\partial \widetilde{z}} \frac{\partial \tilde{u}_{x}}{\partial \widetilde{z}} .
$$

\section{Thermal boundary layer}

Consider again two-dimensional, subsonic, and steady flow over a flat plate. In the framework of boundary-layer theory, energy conservation leads to the following equation for the entropy per mass $s[41]$ :

$$
u_{x} \rho T \frac{\partial s}{\partial x}+u_{z} \rho T \frac{\partial s}{\partial z}=\frac{\partial}{\partial z}\left\{\Lambda \frac{\partial T}{\partial z}\right\} .
$$

Letting $s=s(T, \rho)$, the entropy gradient (say, its $\partial_{z} s$ component) can be expressed as

$$
\frac{\partial s}{\partial z}=\left(\frac{\partial s}{\partial T}\right)_{\rho} \frac{\partial T}{\partial z}+\left(\frac{\partial s}{\partial \rho}\right) \frac{\partial \rho}{\partial z}
$$

The first contribution is directly associated with the isochoric specific heat (per mass) of the gas,

\footnotetext{
${ }^{1}$ Note that the term involving $\tilde{\boldsymbol{\nabla}} \cdot \widetilde{\mathbf{u}}=\partial \widetilde{u}_{x} / \partial \widetilde{x}+\partial \widetilde{u}_{z} / \partial \widetilde{z}$ is of order $1 / \mathrm{Re}$, as long as $\zeta$ and $\eta$ are of the same order of magnitude. Indeed, $\zeta / \eta$ is of order unity if acoustical effects $(\mathrm{Ma} \ll 1)$ and chemical reactions do not take place in the fluid. For situations in which $\zeta$ $>\eta$, see $[[41]$, Sec. 81$]$.
}

$$
T\left(\frac{\partial s}{\partial T}\right)_{\rho} \equiv c_{V} \equiv \frac{c_{P}}{\gamma}
$$

The second contribution follows from a Maxwell relation,

$$
\left(\frac{\partial s}{\partial \rho}\right)_{T}=-\frac{1}{\rho^{2}}\left(\frac{\partial P}{\partial T}\right)_{\rho}=-\frac{1}{\rho T} \frac{c_{P}}{\gamma} \frac{\gamma-1}{\beta} .
$$

Thus, the left-hand side of Eq. (A7) can be written as

$$
\begin{aligned}
u_{x} \rho T \frac{\partial s}{\partial x}+u_{z} \rho T \frac{\partial s}{\partial z}= & \frac{\rho c_{P}}{\gamma}\left\{u_{x} \frac{\partial T}{\partial x}+u_{z} \frac{\partial T}{\partial z}\right\} \\
& -\frac{c_{P}}{\gamma} \frac{\gamma-1}{\beta}\left\{u_{x} \frac{\partial \rho}{\partial x}+u_{z} \frac{\partial \rho}{\partial z}\right\} .
\end{aligned}
$$

Finally, using the continuity equation $u_{x} \partial_{x} \rho+u_{z} \partial_{z} \rho=-\rho\left\{\partial_{x} u_{x}\right.$ $\left.+\partial_{z} u_{z}\right\}$, one finds

$$
u_{x} \frac{\partial T}{\partial x}+u_{z} \frac{\partial T}{\partial z}+\frac{\gamma-1}{\beta}\left\{\frac{\partial u_{x}}{\partial x}+\frac{\partial u_{z}}{\partial z}\right\}=\frac{\gamma}{\rho c_{P}} \frac{\partial}{\partial z}\left\{\Lambda \frac{\partial T}{\partial z}\right\} .
$$

Note that the limiting case of liquids (namely, $\gamma=1$ ) is fully accounted for by this equation.

\section{APPENDIX B: SELF-SIMILARITY ANSATZ}

\section{Viscous boundary layer}

In the stream-function representation (8), the longitudinal velocity $u_{x}$ is expressed as

$$
u_{x}=\frac{1}{\tilde{\rho}} \frac{\partial}{\partial z}\left\{\ell_{c} U_{c} \tilde{\Psi}\right\}=U_{c} \frac{\tilde{\Psi}^{\prime}}{\tilde{\rho}}
$$

and its spatial derivatives are

$$
\begin{gathered}
\frac{\partial u_{x}}{\partial x}=-\frac{\nu_{m}}{2 \ell_{c}^{2}} \frac{\tilde{Z}}{\widetilde{\rho}^{2}}\left\{\tilde{\rho} \tilde{\Psi}^{\prime \prime}-\tilde{\rho}^{\prime} \tilde{\Psi}^{\prime}\right\} \\
\frac{\partial u_{x}}{\partial z}=\frac{U_{c}}{\ell_{c}} \frac{1}{\widetilde{\rho}^{2}}\left\{\widetilde{\rho} \tilde{\Psi}^{\prime \prime}-\tilde{\rho}^{\prime} \tilde{\Psi}^{\prime}\right\} \\
\frac{\partial^{2} u_{x}}{\partial z^{2}}=\frac{U_{c}}{\ell_{c}^{2}} \frac{1}{\widetilde{\rho}^{3}}\left\{\tilde{\rho}^{2} \tilde{\Psi}^{\prime \prime \prime}-2 \tilde{\rho} \tilde{\rho}^{\prime} \tilde{\Psi}^{\prime \prime}+\left[2\left(\tilde{\rho}^{\prime}\right)^{2}-\tilde{\rho} \tilde{\rho}^{\prime \prime}\right] \tilde{\Psi}^{\prime}\right\}
\end{gathered}
$$

Likewise, from Eq. (9), the transverse velocity $u_{z}$ reads

$$
u_{z}=-\frac{1}{\tilde{\rho}} \frac{\partial}{\partial x}\left\{U_{c} \ell_{c} \tilde{\Psi}\right\}=\frac{\nu_{m}}{2 \ell_{c}}\left\{\tilde{Z} \frac{\tilde{\Psi}^{\prime}}{\tilde{\rho}}-\frac{\tilde{\Psi}}{\tilde{\rho}}\right\},
$$

with

$$
\frac{\partial u_{z}}{\partial z}=\frac{\nu_{m}}{2 \ell_{c}^{2}} \frac{1}{\widetilde{\rho}^{2}}\left\{\tilde{Z} \widetilde{\rho} \widetilde{\Psi}^{\prime \prime}-\tilde{Z} \widetilde{\rho}^{\prime} \widetilde{\Psi}^{\prime}+\widetilde{\rho}^{\prime} \widetilde{\Psi}\right\} .
$$

Thus, the advective contributions in Prandtl's equation (5) can be written as

$$
\rho\left\{u_{x} \frac{\partial u_{x}}{\partial x}+u_{z} \frac{\partial u_{x}}{\partial z}\right\}=-\frac{1}{2} \frac{\eta_{m} U_{c}}{\tilde{\rho} \ell_{c}^{2}}\left\{\tilde{\Psi} \tilde{\Psi}^{\prime \prime}-\frac{\tilde{\rho}^{\prime}}{\tilde{\rho}} \tilde{\Psi} \tilde{\Psi}^{\prime}\right\}
$$


TABLE III. Simulation results of the relative deviation of the Nusselt number $\mathrm{Nu} / \mathrm{Nu}_{\mathrm{OB}}-1$ at the temperature difference of $\Delta=10 \mathrm{~K}$ for several hypothetical fluids. At the Rayleigh numbers of $\mathrm{Ra}=10^{6}, 10^{7}$, and $10^{8}$ the $\mathrm{OB}$ Nusselt numbers correspond to $\mathrm{Nu}_{\mathrm{OB}}=6.53,12.43$, and 25.12, respectively. The notations for the material properties are the same as in Table II.

\begin{tabular}{|c|c|c|c|c|c|c|}
\hline Case & $\hat{\beta}$ & $\kappa$ & $\nu$ & $\begin{array}{c}100\left(\mathrm{Nu} / \mathrm{Nu}_{\mathrm{OB}}-1\right) \\
\text { at } \mathrm{Ra}=10^{6}\end{array}$ & $\begin{array}{c}100\left(\mathrm{Nu} / \mathrm{Nu}_{\mathrm{OB}}-1\right) \\
\text { at } \mathrm{Ra}=10^{7}\end{array}$ & $\begin{array}{c}100\left(\mathrm{Nu} / \mathrm{Nu}_{\mathrm{OB}}-1\right) \\
\text { at } \mathrm{Ra}=10^{8}\end{array}$ \\
\hline $1(\mathrm{NOB})$ & $\hat{\beta}(T)$ & $\kappa(T)$ & $\nu(T)$ & $0.7097 \pm 0.1517$ & $0.8956 \pm 0.2213$ & $1.2403 \pm 0.2316$ \\
\hline 2 & $\hat{\beta}(T)$ & $\kappa(T)$ & $\nu_{m}$ & $0.1655 \pm 0.1511$ & $0.4795 \pm 0.2323$ & $0.4040 \pm 0.2328$ \\
\hline 3 & $\hat{\beta}(T)$ & $\kappa_{m}$ & $\nu(T)$ & $1.3160 \pm 0.1518$ & $1.5812 \pm 0.2230$ & $1.6502 \pm 0.2234$ \\
\hline 4 & $\hat{\beta}(T)$ & $\kappa_{m}$ & $\nu_{m}$ & $0.6640 \pm 0.1505$ & $0.4963 \pm 0.2324$ & $0.6450 \pm 0.2425$ \\
\hline 5 & $\hat{\beta}\left(2 T_{m}-T\right)$ & $\kappa(T)$ & $\nu(T)$ & $1.0087 \pm 0.1518$ & $1.2804 \pm 0.2214$ & $1.3223 \pm 0.2228$ \\
\hline 6 & $\hat{\beta}\left(2 T_{m}-T\right)$ & $\kappa(T)$ & $\nu_{m}$ & $0.1655 \pm 0.1511$ & $0.4795 \pm 0.2323$ & $0.4040 \pm 0.2328$ \\
\hline 7 & $\hat{\beta}\left(2 T_{m}-T\right)$ & $\kappa_{m}$ & $\nu(T)$ & $0.9925 \pm 0.1518$ & $1.1741 \pm 0.2255$ & $1.0832 \pm 0.2329$ \\
\hline 8 & $\hat{\beta}\left(2 T_{m}-T\right)$ & $\kappa_{m}$ & $\nu_{m}$ & $0.5988 \pm 0.1505$ & $0.9991 \pm 0.2236$ & $1.0073 \pm 0.2286$ \\
\hline 9 & $\beta_{m}$ & $\kappa(T)$ & $\nu(T)$ & $0.3425 \pm 0.1517$ & $0.6188 \pm 0.2175$ & $0.0538 \pm 0.2455$ \\
\hline 10 & $\beta_{m}$ & $\kappa(T)$ & $\nu_{m}$ & $-0.1734 \pm 0.1509$ & $-0.4404 \pm 0.2277$ & $0.0195 \pm 0.2246$ \\
\hline 11 & $\beta_{m}$ & $\kappa_{m}$ & $\nu(T)$ & $0.6380 \pm 0.1523$ & $0.4491 \pm 0.2321$ & $0.7775 \pm 0.2222$ \\
\hline $12(\mathrm{OB})$ & $\beta_{m}$ & $\kappa_{m}$ & $\nu_{m}$ & $0.0000 \pm 0.1070$ & $0.0000 \pm 0.1573$ & $0.0000 \pm 0.1689$ \\
\hline
\end{tabular}

Now consider the viscous contributions:

$$
\frac{\partial}{\partial z}\left\{\eta \frac{\partial u_{x}}{\partial z}\right\}=\eta \frac{\partial^{2} u_{x}}{\partial z^{2}}+\frac{\partial \eta}{\partial z} \frac{\partial u_{x}}{\partial z} .
$$

Since

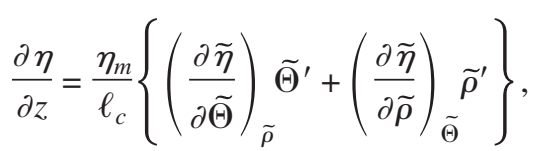

insertion of (B2) and (B3) into (B6) leads to

$$
\begin{aligned}
\frac{\widetilde{\rho} \ell_{c}^{2}}{\eta_{m} U_{c}} \frac{\partial}{\partial z}\left\{\eta \frac{\partial u_{x}}{\partial z}\right\}= & \widetilde{\eta} \widetilde{\Psi}^{\prime \prime \prime}+\left(\widetilde{\eta}^{\prime}-2 \frac{\widetilde{\rho}^{\prime}}{\tilde{\rho}} \tilde{\eta}\right) \widetilde{\Psi}^{\prime \prime}+\left\{\left[2\left(\frac{\widetilde{\rho}^{\prime}}{\tilde{\rho}}\right)^{2}\right.\right. \\
& \left.\left.-\frac{\widetilde{\rho}^{\prime \prime}}{\widetilde{\rho}}\right] \tilde{\eta}-\frac{\widetilde{\rho}^{\prime}}{\widetilde{\rho}} \widetilde{\eta}^{\prime}\right\} \widetilde{\Psi}^{\prime} .
\end{aligned}
$$

Therefore, by substituting (B5) and (B8) into (5) one finally obtains Eq. (11).

\section{Thermal boundary layer}

In the same spirit as above, inserting (B1) and (B4), and

$$
\frac{\partial}{\partial z}\left\{\Lambda \frac{\partial T}{\partial z}\right\}=\frac{\Lambda_{m} \Delta}{\ell_{c}^{2}}\left\{\tilde{\Lambda} \widetilde{\Theta}^{\prime \prime}+\tilde{\Lambda}^{\prime} \widetilde{\Theta}^{\prime}\right\}
$$

into Eq. (6), one finds

$$
-\frac{\nu_{m} \Delta}{2 \ell_{c}^{2}} \frac{\widetilde{\Psi}}{\tilde{\rho}} \widetilde{\Theta}^{\prime}+\frac{\nu_{m}}{2 \ell_{c}^{2}} \frac{\gamma-1}{\beta} \frac{\widetilde{\rho}^{\prime}}{\widetilde{\rho}^{2}} \widetilde{\Psi}=\frac{\gamma}{\tilde{\rho} \widetilde{c}_{P}} \frac{1}{\operatorname{Pr}}\left\{\tilde{\Lambda} \widetilde{\Theta}^{\prime \prime}+\widetilde{\Lambda}^{\prime} \widetilde{\Theta}^{\prime}\right\},
$$

where $\widetilde{\beta}=\beta \Delta$ and $\operatorname{Pr}=\left(\nu_{m} \rho_{m} c_{P, m}\right) / \Lambda_{m}$. Thus

$$
\widetilde{\Lambda} \widetilde{\Theta}^{\prime \prime}+\left\{\frac{1}{2} \frac{\widetilde{c}_{P}}{\gamma} \operatorname{Pr} \widetilde{\Psi}+\widetilde{\Lambda}^{\prime}\right\} \widetilde{\Theta}^{\prime}-\frac{\gamma-1}{2 \gamma} \frac{\widetilde{c}_{P}}{\widetilde{\beta}} \frac{\widetilde{\rho}^{\prime}}{\widetilde{\rho}} \operatorname{Pr} \widetilde{\Psi}=0 .
$$

Here, substituting $\widetilde{\rho}^{\prime}$ by (12) one finally obtains

$$
\widetilde{\Lambda} \widetilde{\Theta}^{\prime \prime}+\left\{\frac{1}{2} \widetilde{c}_{P} \operatorname{Pr} \widetilde{\Psi}+\widetilde{\Lambda}^{\prime}\right\} \widetilde{\Theta}^{\prime}=0 .
$$

Note that the limiting case of liquids (namely, $\gamma=1$ ) is fully accounted for by this equation.

\section{APPENDIX C: NUMERICAL RESULTS ON NUSSELT NUMBERS IN REAL AND HYPOTHETICAL ETHANE FLUIDS}

For completeness, in Table III the NOB corrections in the Nusselt number are given, resulting from the numerical simulations of real and hypothetical ethane. The corresponding NOB corrections of the center temperature were already shown in Table II. 
[1] B. Castaing, G. Gunaratne, F. Heslot, L. Kadanoff, A. Libchaber, S. Thomae, X. Z. Wu, S. Zaleski, and G. Zanetti, J. Fluid Mech. 204, 1 (1989).

[2] E. D. Siggia, Annu. Rev. Fluid Mech. 26, 137 (1994).

[3] S. Cioni, S. Ciliberto, and J. Sommeria, J. Fluid Mech. 335, 111 (1997).

[4] X. Chavanne, F. Chilla, B. Castaing, B. Hebral, B. Chabaud, and J. Chaussy, Phys. Rev. Lett. 79, 3648 (1997).

[5] X. Xu, K. M. S. Bajaj, and G. Ahlers, Phys. Rev. Lett. 84, 4357 (2000).

[6] J. Niemela, L. Skrebek, K. R. Sreenivasan, and R. Donnelly, Nature (London) 404, 837 (2000).

[7] X. Chavanne, F. Chilla, B. Chabaud, B. Castaing, and B. Hebral, Phys. Fluids 13, 1300 (2001).

[8] G. Ahlers and X. Xu, Phys. Rev. Lett. 86, 3320 (2001).

[9] X. L. Qiu and P. Tong, Phys. Rev. E 64, 036304 (2001).

[10] L. P. Kadanoff, Phys. Today 54 (8), 34 (2001).

[11] K.-Q. Xia, S. Lam, and S. Q. Zhou, Phys. Rev. Lett. 88, 064501 (2002).

[12] P. E. Roche, B. Castaing, B. Chabaud, and B. Hebral, Europhys. Lett. 58, 693 (2002).

[13] J. Niemela and K. R. Sreenivasan, J. Fluid Mech. 481, 355 (2003).

[14] D. Funfschilling and G. Ahlers, Phys. Rev. Lett. 92, 194502 (2004).

[15] E. Brown, A. Nikolaenko, and G. Ahlers, Phys. Rev. Lett. 95, 084503 (2005).

[16] A. Nikolaenko, E. Brown, D. Funfschilling, and G. Ahlers, J. Fluid Mech. 523, 251 (2005).

[17] J. Niemela and K. R. Sreenivasan, J. Fluid Mech. 557, 411 (2006).

[18] K.-Q. Xia, C. Sun, and S. Q. Zhou, Phys. Rev. E 68, 066303 (2003).

[19] X. D. Shang, X. L. Qiu, P. Tong, and K.-Q. Xia, Phys. Rev. Lett. 90, 074501 (2003).

[20] P. E. Roche, B. Castaing, B. Chabaud, and B. Hebral, J. Low Temp. Phys. 134, 1011 (2004).

[21] C. Sun, K. Q. Xia, and P. Tong, Phys. Rev. E 72, 026302 (2005).

[22] E. Brown and G. Ahlers, J. Fluid Mech. 568, 351 (2006).

[23] J. Niemela and K. R. Sreenivasan, J. Low Temp. Phys. 143, 163 (2006).

[24] R. du Puits, C. Resagk, A. Tilgner, F. H. Busse, and A. Thess, J. Fluid Mech. 572, 231 (2007).

[25] R. Kerr, J. Fluid Mech. 310, 139 (1996).

[26] R. Benzi, F. Toschi, and R. Tripiccione, J. Stat. Phys. 93, 901 (1998).

[27] R. Kerr and J. R. Herring, J. Fluid Mech. 419, 325 (2000).

[28] R. Verzicco and R. Camussi, J. Fluid Mech. 383, 55 (1999).

[29] R. Verzicco and R. Camussi, J. Fluid Mech. 477, 19 (2003).

[30] D. Lohse and F. Toschi, Phys. Rev. Lett. 90, 034502 (2003).

[31] R. Verzicco, Phys. Fluids 16, 1965 (2004).

[32] G. Amati, K. Koal, F. Massaioli, K. R. Sreenivasan, and R.
Verzicco, Phys. Fluids 17, 121701 (2005).

[33] O. Shishkina and C. Wagner, J. Fluid Mech. 546, 51 (2006).

[34] G. Stringano and R. Verzicco, J. Fluid Mech. 548, 1 (2006).

[35] R. P. J. Kunnen, H. J. H. Clercx, B. J. Geurts, L. J. A. van Bokhoven, R. A. D. Akkermans, and R. Verzicco, Phys. Rev. E 77, 016302 (2008).

[36] S. Grossmann and D. Lohse, J. Fluid Mech. 407, 27 (2000); Phys. Rev. Lett. 86, 3316 (2001); Phys. Rev. E 66, 016305 (2002); Phys. Fluids 16, 4462 (2004).

[37] R. Benzi, Phys. Rev. Lett. 95, 024502 (2005).

[38] E. Brown and G. Ahlers, Phys. Rev. Lett. 98, 134501 (2007).

[39] A. Oberbeck, Ann. Phys. Chem. 243, 243 (1879).

[40] J. Boussinesq, Theorie Analytique de la Chaleur (GauthierVillars, Paris, 1903), Vol. 2.

[41] L. D. Landau and E. M. Lifshitz, Fluid Mechanics (Pergamon Press, Oxford, 1987).

[42] S. Chandrasekhar, Hydrodynamic and Hydromagnetic Stability (Dover, New York, 1981).

[43] G. Ahlers, E. Brown, F. Fontenele Araujo, D. Funfschilling, S. Grossmann, and D. Lohse, J. Fluid Mech. 569, 409 (2006).

[44] J. Zhang, S. Childress, and A. Libchaber, Phys. Fluids 9, 1034 (1997).

[45] K. Sugiyama, E. Calzavarini, S. Grossmann, and D. Lohse, Europhys. Lett. 80, 34002 (2007).

[46] G. Ahlers, F. Fontenele Araujo, D. Funfschilling, S. Grossmann, and D. Lohse, Phys. Rev. Lett. 98, 054501 (2007).

[47] D. G. Friend, H. Ingham, and J. F. Ely, J. Phys. Chem. Ref. Data 20, 275 (1991).

[48] S. Ciliberto, S. Cioni, and C. Laroche, Phys. Rev. E 54, R5901 (1996).

[49] G. Ahlers, E. Brown, and A. Nikolaenko, J. Fluid Mech. 557, 347 (2006).

[50] H. Schlichting and K. Gersten, Boundary Layer Theory, 8th ed. (Springer Verlag, Berlin, 2000).

[51] K. Stewartson, The Theory of Laminar Boundary Layers in Compressible Fluids (Oxford University Press, Oxford, 1964).

[52] R. Krishnamurti and L. N. Howard, Proc. Natl. Acad. Sci. U.S.A. 78, 1981 (1981).

[53] W. Press, S. Teukolsky, W. Vetterling, and B. Flannery, Numerical Recipes (Cambridge University Press, Cambridge, U.K., 1986).

[54] G. Ahlers, D. S. Cannell, L. I. Berge, and S. Sakurai, Phys. Rev. E 49, 545 (1994).

[55] K. Müller, G. Ahlers, and F. Pobell, Phys. Rev. B 14, 2096 (1976).

[56] G. Ahlers, Phys. Rev. E 63, 015303(R) (2000).

[57] P. Roche, B. Castaing, B. Chabaud, B. Hebral, and J. Sommeria, Eur. Phys. J. B 24, 405 (2001).

[58] E. Brown, D. Funfschilling, A. Nikolaenko, and G. Ahlers, Phys. Fluids 17, 075108 (2005).

[59] E. Brown and G. Ahlers, Europhys. Lett. 80, 14001 (2007).

[60] K. Sugiyama, E. Calzavarini, S. Grossmann, and D. Lohse (unpublished). 